\title{
Oil and Gas Pipeline Construction-Induced Forest Fragmentation and Biodiversity Loss in the Niger Delta, Nigeria
}

\author{
Ikechukwu O. Agbagwa*, Benjamin C. Ndukwu \\ Department of Plant Science and Biotechnology, Faculty of Biological Sciences, University of Port Harcourt, \\ Port Harcourt, Nigeria \\ Email: ${ }^{*}$ ikechukwu.agbagwa@gmail.com, ${ }^{*}$ ikechukwu.agbagwa@uniport.edu.ng
}

Received 17 July 2014; revised 3 August 2014; accepted 14 August 2014

Copyright $(\underset{2014}{2}$ by authors and Scientific Research Publishing Inc.

This work is licensed under the Creative Commons Attribution International License (CC BY). http://creativecommons.org/licenses/by/4.0/

(c) (i) Open Access

\section{Abstract}

The impacts of oil and gas pipeline construction on the forest and biodiversity in parts of the Niger Delta was investigated. This was achieved by studying the construction activities and operational phases of two pipelines with cumulative length of $165 \mathrm{~km}$ vis-à-vis the biodiversity of the affected area. The study involved field sampling along the RoWs to inventorize the fauna and flora, visual assessments and interview with locals. It was observed that the pipelines traversed moist lowland/freshwater swamp and mangrove forests, and barrier islands with approximately 4,950,000 $\mathrm{m}^{2}$ (equivalent of 495 hectares) of forest cleared and 9,642,000 trees killed to realize the pipelines. A total of 219 plant species in 66 families and 125 different fauna species from 64 families were recorded in these areas. Three out of the 4 recorded species of Meliaceae were threatened based on IUCN Conservation Status. Also based on IUCN Conservation Status, 20 mammals, including Pan troglodytes, Cercopithecus erythrogaster and Trichechus senegalensis, 7 birds, 2 reptiles and 1 amphibian were within threatened group. The results showed that the study area had witnessed colossal loss of biodiversity due to habitat displacement, forest fragmentation and deforestation, and escalated exploitation of species. The study identified the most affected biodiversity, and proffered measures to mitigate such occurrences.

\section{Keywords}

Biodiversity, Forest Fragmentation, Pipeline Construction, Right of Way, Niger Delta

\footnotetext{
*Corresponding author.
}

How to cite this paper: Agbagwa, I.O. and Ndukwu, B.C. (2014) Oil and Gas Pipeline Construction-Induced Forest Fragmentation and Biodiversity Loss in the Niger Delta, Nigeria. Natural Resources, 5, 698-718. 


\section{Introduction}

The Niger Delta covers an area of approximately $50,000 \mathrm{~km}^{2}$ between Latitudes $4^{\circ} 0^{\prime} 0^{\prime \prime} \mathrm{N}$ and $8^{\circ} 0^{\prime} 0^{\prime \prime} \mathrm{N}$, and Longitudes $5^{\circ} 0^{\prime} 0$ "E and $7^{\circ} 0^{\prime} 0 " \mathrm{E}$ [1] (Figure 1). It is within two meters above sea level [2] and extends over four ecological zones: coastal barrier islands, brackish/saline water mangrove swamps, freshwater swamp forests (permanent and seasonal), and dry upper plain lowland rain forests [3]. The hydrological boundaries between these ecological zones are imperceptible; so also are some of the forest zones particularly the fringing of the mangrove forest and freshwater swamp forest. It is also the largest wetland in Africa with rich biological diversity [1] [4]-[8]. Its mangrove forest is the largest in Africa $\left(11,134 \mathrm{~km}^{2}\right)$ and the fourth largest in the world [9].

Across the different ecological zones, the Niger Delta is home to diverse assemblages of Not Evaluated (NE), Data Deficient (DD), Least Concern (LC), Near Threatened (NT), Vulnerable (VU), Endangered (EN) and Critically Endangered (CR) wildlife species. Some of the threatened species, which have been previously reported in IUCN red list are the pygmy hippopotamus (Choeropsis liberiensis), manatees (Trichechus senegalensis), maritime hippopotamus (Hippopotamus amphibious), Nile crocodile (Crocodylus niloticus), slender nosed crocodile (Crocodylus cataphractus) and dwarf crocodile (Osteolaemus tetraspis) [3] [4]. Ohimain [5] also reported that such wildlife species as the Cape clawless otter (Aonyx capensis), African palm nut vulture (Gypohierax angolensis), fire-footed squirrel (Funisciurus pyrropus), Hammerkop (Scopus umbretta), African fish eagle (Haliaeetus vocifer), Sclater's guenon (Cercopithecus sclateri), sitatunga (Tragelaphus spekei), white throated monkey (Cercopithecus erythrogaster), which occurred in the area required conservation.

However, the Niger Delta is known and recognized globally not for the rich biological diversity but for its non-renewable oil and gas resources. The region is home to the hydrocarbon exploration and production activities in Nigeria where proceeds from this singular industry (i.e., oil and gas) accounts for over $90 \%$ of the nation's total export earnings [1]. With proven crude oil reserve of 37.2 billion barrels, 165 trillion standard cubic feet (scf) of natural gas, 75.4 trillion scf of non associated gas and average of 2.28 million barrels of crude oil production per day, Nigeria is Africa's largest crude oil producer, the world's 11th largest producer as well as 8th largest exporter 2011 [10]. Nigeria has been exporting crude oil from this region since 1958. Effective oil and gas exploration and production has therefore taken place in this region described as environmentally sensitive

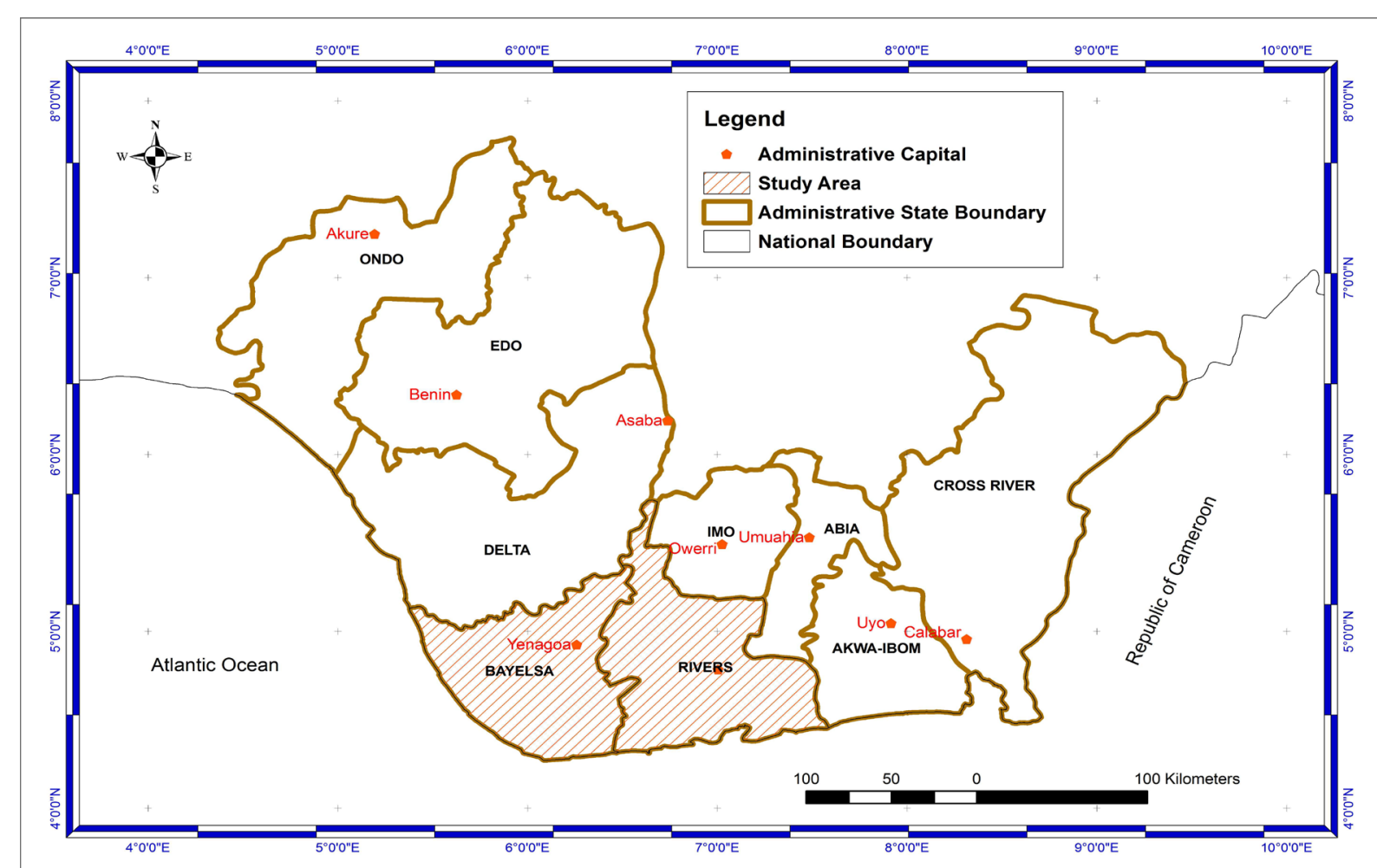

Figure 1. Map showing the Niger Delta of Nigeria. 
and fragile [1] [3], with brackish and fresh water swamps cris-crossed by anastomosing network of rivers, creeks and distributaries/tidal channels [7] for more than fifty years. This will likely continue for the next fifty years or more.

The completion of every successful oil and gas exploration and production activity is to hook up the producing well to a gathering facility through a flowline. The gathering facility may be a flow station or Floating Production Storage and Offloading (FPSO) system (strictly offshore). These products are transported from the gathering facility to a refinery or outright selling point (terminals) through pipelines. In the Niger Delta the flowlines and pipelines range from diameter of six inches (6") to thirty six inches (36") with Right of Way (RoW) of $15 \mathrm{~m}$ to $30 \mathrm{~m}$ in width. Their construction involves acquisition and clearing the RoW of all vegetation and wildlife, and excavation by the open-cut method (thrust boring at major road and river crossings). The open cut method requires deployment of excavators and other earth-moving equipment: the depth of excavation ranging from $3 \mathrm{~m}$ to $15 \mathrm{~m}$. Backfilling after pipe laying is mostly haphazard resulting in blockage of natural drainages and eventual flooding [6] [7]. At present, there are 23 export terminals, 112 flow stations and over 20,000 $\mathrm{km}$ of flowline/pipeline infrastructure concentrated within this $50,000 \mathrm{~km}^{2}$ area.

Studies and reports implicating different oil and gas exploration and production activities in the Niger Delta to forest fragmentation and overall biodiversity depletion abound [1] [3]-[7] [11]-[18]. Empirical investigations into flowline/pipeline construction activities and their operation vis-à-vis their impacts on this ecologically and biologically diverse but fragile and sensitive region are lacking. This study investigates and quantifies the impact of flowline/pipeline construction activities in the Niger Delta and proffers remedial measures to mitigate and restore adversely impacted areas.

\section{Materials and Methods}

\subsection{Study Area and Rationale}

The field studies were conducted between 2009 and 2012 in Rivers and Bayelsa States within latitudes $4^{\circ} 0^{\prime} 0^{\prime \prime} \mathrm{N}$ and $6^{\circ} 0^{\prime} 0^{\prime \prime} \mathrm{N}$, and longitudes $5^{\circ} 0^{\prime} 0^{\prime \prime} \mathrm{E}$ and $7^{\circ} 0^{\prime} 0^{\prime \prime} \mathrm{E}$ (Figure 1). The studies were tailored to capture two broad categories of oil and gas pipeline impacts on biodiversity (i.e., plants and wildlife) viz: (i) impacts associated with pipeline construction and (ii) impacts associated with the operational phase of pipeline facilities. To achieve these objectives, two different pipeline RoWs at different stages of existence were chosen.

1) A $45 \mathrm{~km}$ pipeline project under construction (Figure 2(A)). This is within lowland and freshwater swamp forests. RoW clearing and excavation/trenching activities were observed vis-à-vis impacts on biodiversity.

2) A $120 \mathrm{~km}$ long pipeline (Figures 2(B)-(D)), which has been in existence for upwards of twenty five (25) years. The pipeline originated in a lowland rainforest, crossed seasonal and permanent freshwater swamp forests, mangrove forests and terminated in a barrier island. During the study, a section of this pipeline within the mangrove forest section was being replaced. This replacement activity increased the opportunity to document construction impact on the mangrove forest area.

Study methodology included inventory/sampling of plant and animal species, observations and use of still life photographs and interview/discussions with locals; comparison with forest climaxes in contiguous areas and by copious reference to existing information [9]. For vegetation and wildlife assessments, the pipeline RoWs provided continuous linear transect.

\subsection{Vegetation Sampling and Assessment}

At each sampling point along the transect, a tape measure was used to mark out $100 \mathrm{~m} \times 100 \mathrm{~m}$ plot. Each 100 $\mathrm{m} \times 100 \mathrm{~m}$ plot was further demarcated into four random blocks of $20 \mathrm{~m} \times 20 \mathrm{~m}$. This was to ease sampling and increase the opportunity of encountering all plant species during inventory. Within each chosen block, vegetation assessment was made through copious visual observations of the status of the flora. On-site identification as well as inventory of all plant species within each sample station was carried out. Data obtained was further used for determining the density, relative distribution and frequency of occurrence of major plant species. The structure and physiognomy of the vegetation of the area [height, crown diameter and crown depth of all woody stems $3 \mathrm{~m}$ or more in height or over $10 \mathrm{~cm}$ in diameter at breast height $(1.3 \mathrm{~m})]$ were estimated in the field and ratified with reference to Richards [19]. Trees were identified to species level using Flora of Tropical West Africa [20]-[22] and Trees of Nigeria [23]. 

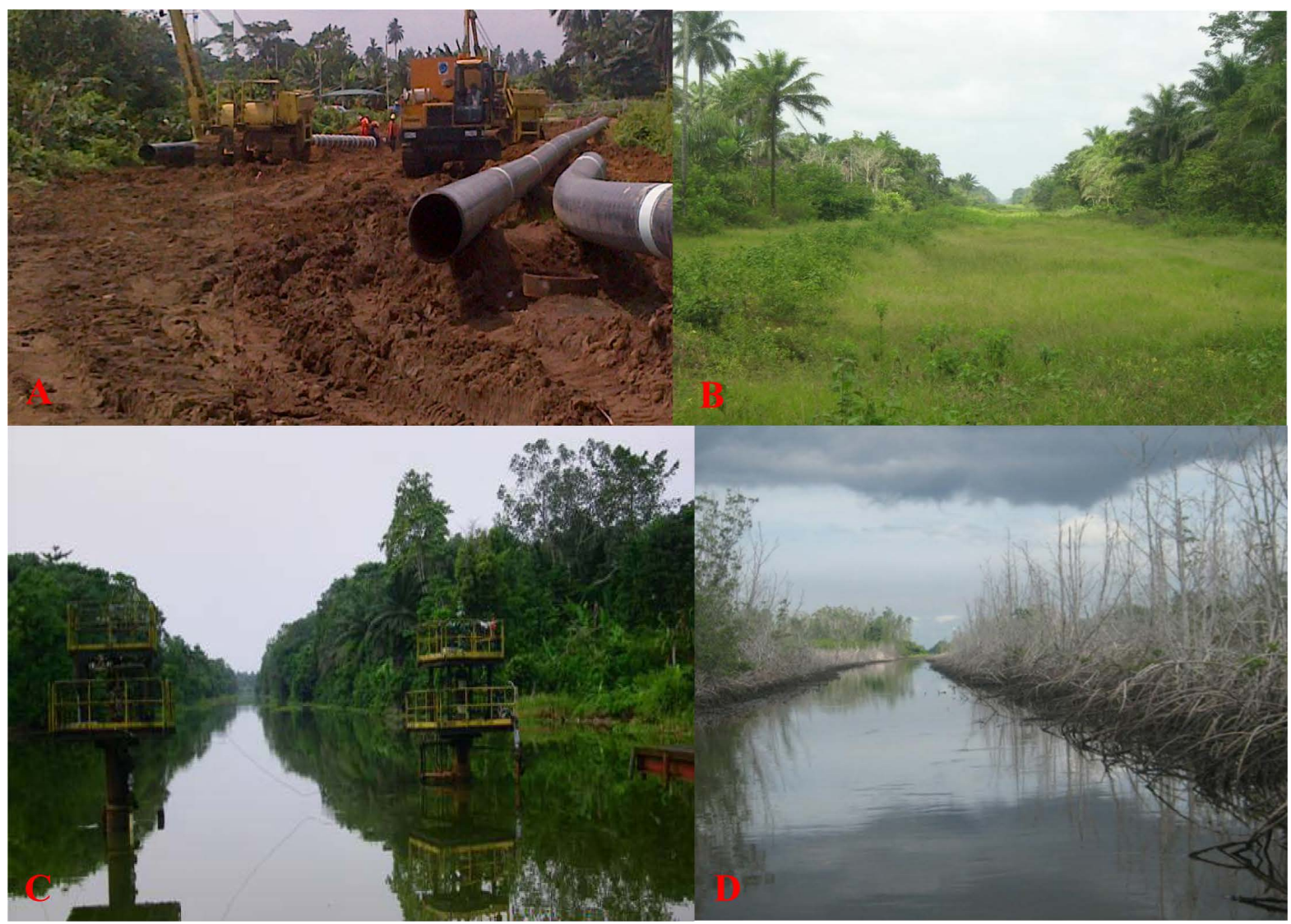

Figure 2. (A) Section of the $45 \mathrm{~km}$ pipeline under construction in moist lowland rainforest section. Notice the different earth moving equipment, excavated earth, pipes; (B)-(D) Different segments of the $120 \mathrm{~km}$ existing pipeline RoW ((B) is along lowland rainforest area, $(\mathrm{C})$ is across freshwater swamp forest area, and (D) is through mangrove swamp forest i.e., brackish water area).

\subsection{Wildlife Survey and Assessment}

The wildlife assessment involved a survey of amphibians, reptiles, birds, and mammals in the study areas using the existing RoW as linear transects. For the census of the birds, reptiles and other animals, which readily offered themselves for observation, the direct count method was adopted using a pair of binoculars. By probing such humid habitats as logs, heaps of dry or decaying leaves, ponds, forest undergrowth and burrows, the presence of some animals like amphibians, reptiles and small mammals were revealed. All captured, dislodged or sighted animals were identified, often on the spot, to possible taxonomic levels using field guides and keys [24]-[28]. For species which do not offer themselves readily for observation, the indirect method, which makes use of evidence of animal's presence [29], was used. Such signs of animal occupation as burrows, faecal pellets (droppings), hairs, foot prints or tracks, sloughed skin, devoured food (cassava, yam tubers, oil-palm fruits, other fruits), as well as vocalization, skeleton/carcass and trampled grass were of immense use in the course of the investigation. Further information on the wildlife diversity, abundance and use in the areas were obtained through inspecting series of traps and snares set by hunters and interviewing them. Wildlife species displayed for sale around villages close to the RoWs were noted.

\section{Results}

\subsection{Status of the Forest and Vegetation of the Area}

The $165 \mathrm{~km}$ study area comprises $133 \mathrm{~km}$ of moist lowland/freshwater swamp and $32 \mathrm{~km}$ of mangrove swamp forests. Typically, the pipeline RoWs have width of $30 \mathrm{~m}$. Thus, approximately 4,950,000 $\mathrm{m}^{2}$ (equivalent of 495 hectares) area of moist lowland/freshwater swamp forest $\left(3,990,000 \mathrm{~m}^{2} \approx 399\right.$ hectares from $133 \mathrm{~km}$ long RoW) 
and mangrove swamp forest $\left(960,000 \mathrm{~m}^{2} \approx 96\right.$ hectares for $\left.32 \mathrm{~km}\right)$ was cleared to realize the pipelines. Observations and results from the study show that the area has different forest ecosystems, which are rich in biological diversity. A total of 219 species (192 in moist lowland rainforest, 113 in freshwater swamp forest and 8 in mangrove swamp forest) of plants from 66 different families were recorded (Table 1). The family Leguminosae

\section{Table 1. List of plant species showing their distribution/occurrence in the different forest types.}

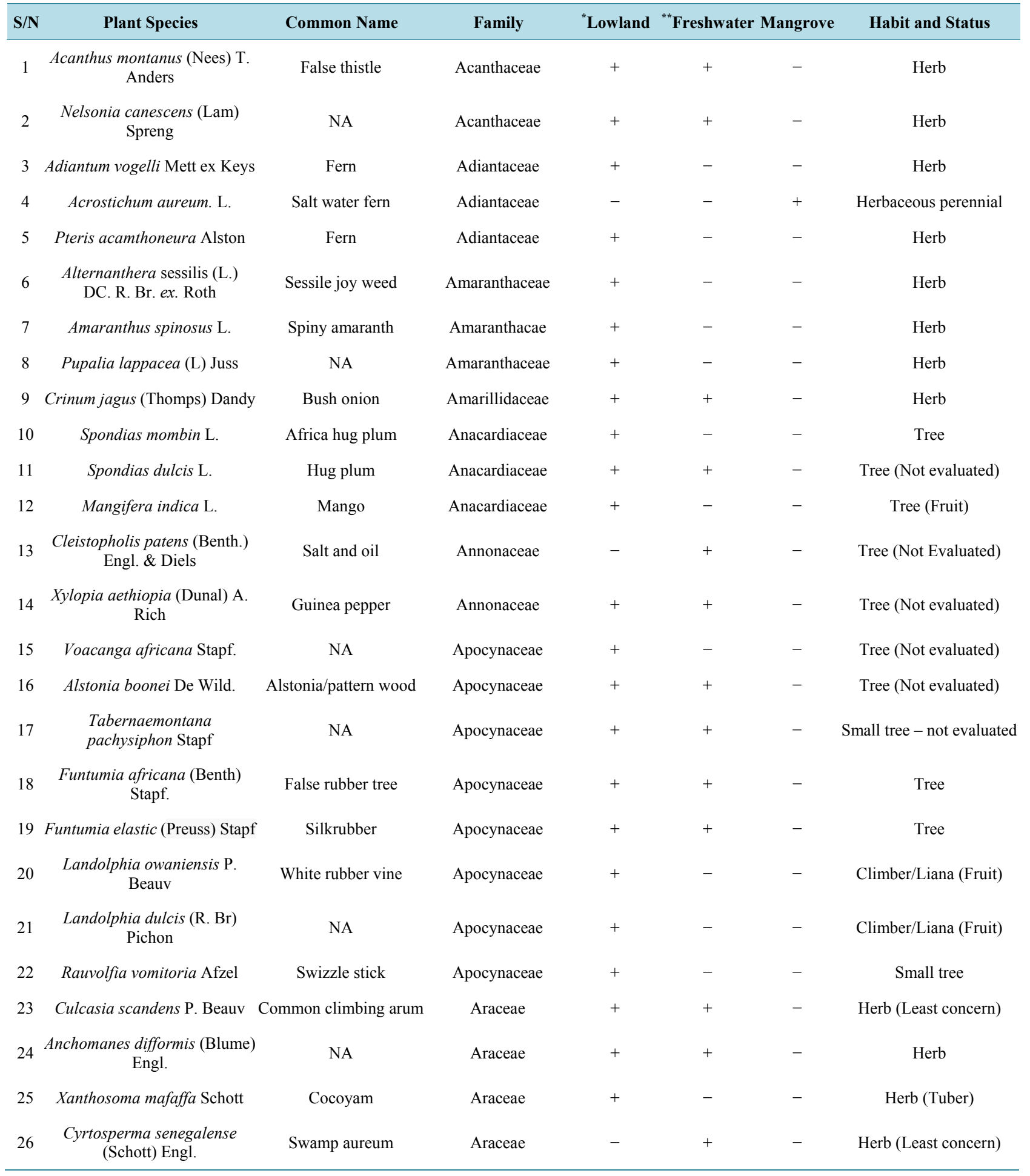




\begin{tabular}{|c|c|c|c|c|c|c|c|}
\hline 27 & Cocos nucifera Linn & Coconut & Arecaceae & + & + & - & Tree (Fruit) \\
\hline 28 & $\begin{array}{l}\text { Ancistrophyllum secundiflorum } \\
\text { (P. Beauv) Wendl. }\end{array}$ & Climbing palm & Arecaceae & + & + & - & Climber/Liana \\
\hline 29 & $\begin{array}{l}\text { Raphia hookeri G. Mann \& H. } \\
\text { Wendl. }\end{array}$ & Raffia palm & Arecaceae & - & + & - & Tree \\
\hline 30 & Elaeis guineensis Jacq & Oil palm & Arecaceae & + & + & - & Tree \\
\hline 31 & $\begin{array}{l}\text { Laccosperma arcutiflora } \\
\text { (Becc.) J. Dransf }\end{array}$ & NA & Arecaceae & + & + & - & Shrub \\
\hline 32 & $\begin{array}{l}\text { Laccosperma opacum } \\
\text { (G. Mann \& H.Wendl) Drude }\end{array}$ & Oga plant & Arecaceae & + & + & - & Shrub \\
\hline 33 & Borassus aethiopum Mart & African fan palm & Arecaceae & + & - & - & Tree \\
\hline 34 & Nypa fruticans Wumb. & Nypa palm & Arecaceae & - & - & + & Shrub (Least concern) \\
\hline 35 & Daemia extensa (Forssk) Chev & NA & Asclepiadaceae & + & - & - & Climber \\
\hline 36 & $\begin{array}{c}\text { Chromolaena odorata }(\text { Linn) } \\
\text { King \& Robinson }\end{array}$ & Siamweed & Asteraceae & + & - & - & Shrub \\
\hline 37 & $\begin{array}{l}\text { Conyza sumatrensis } \\
\text { (Retz) E.H. Walker }\end{array}$ & NA & Asteraceae & + & - & - & Herb \\
\hline 38 & $\begin{array}{l}\text { Synedrella nodiflora }(\mathrm{L} .) \\
\text { Gaertn }\end{array}$ & Nodeweed & Asteraceae & + & - & - & Herb \\
\hline 39 & $\begin{array}{l}\text { Aspilia africana (Pers) } \\
\text { C.D. Adams }\end{array}$ & Haemorhage plant & Asteraceae & + & - & - & Herb \\
\hline 40 & $\begin{array}{c}\text { Mikania cordata (Burm F.) } \\
\text { Robinson }\end{array}$ & Climbing hemp weed & Asteraceae & + & + & - & Climber \\
\hline 41 & Vernonia amygdalina Del & Bitter leaf & Asteraceae & + & - & - & Shrub \\
\hline 42 & Ageratum conyzoides $\mathrm{L}$. & Goat weed & Asteraceae & + & - & - & Herb \\
\hline 43 & $\begin{array}{c}\text { Diplazium sammatii (Kuhn) } \\
\text { C. Chr. }\end{array}$ & NA & Athyraceae & - & + & - & Herb \\
\hline 44 & Avicenia africana P. Beauv. & White mangrove & Avicenaceae & - & - & + & $\begin{array}{c}\text { Tree } \\
\text { (Not evaluated) }\end{array}$ \\
\hline 45 & $\begin{array}{c}\text { Kigelia africana (Lam.) } \\
\text { Beneth. }\end{array}$ & Sausage plant & Bignoniaceae & + & + & - & $\begin{array}{c}\text { Tree } \\
\text { (Not evaluated) }\end{array}$ \\
\hline 46 & Newbouldia laevis Seem & African Border tree & Bignoniaceae & + & - & - & Tree \\
\hline 47 & Ceiba pentandra (L.) Gaertn & Cotton wood tree & Bombacaceae & + & + & - & $\begin{array}{c}\text { Tree } \\
\text { (Not evaluated) }\end{array}$ \\
\hline 48 & $\begin{array}{c}\text { Bombax buonopozense } \mathrm{P} . \\
\text { Beauv. }\end{array}$ & Silt cotton tree & Bombacaceae & + & + & - & Tree (VU) \\
\hline 49 & Heliotropum indicum $\mathrm{L}$. & Cock's comb & Boraginaceae & + & + & - & Herb \\
\hline 50 & Canarium schweinfurthii Engl. & NA & Burseraceae & + & + & - & Tree \\
\hline 51 & $\begin{array}{c}\text { Dacryodes edulis (G. Don) } \\
\text { H.J. Lam }\end{array}$ & Native pear & Burseraceae & + & - & - & Tree \\
\hline 52 & Carica papaya $\mathrm{L}$. & Pawpaw & Caricaceae & + & - & - & Tree \\
\hline 53 & Musanga cecropoides $\mathrm{R}$. Br. & Umbrella tree & Cecropiaceae & + & + & - & Tree \\
\hline 54 & $\begin{array}{l}\text { Chrysobalanus orbicularis } \\
\text { Schumach. }\end{array}$ & NA & Chrysobalanaceae & + & - & - & Shrub \\
\hline 55 & $\begin{array}{c}\text { Combetum zenkeri Engl. \& } \\
\text { Diels }\end{array}$ & NA & Combretaceae & + & - & - & Climber \\
\hline 56 & Terminalia catappa $\mathrm{L}$. & Almond Indian fruit & Combretaceae & + & - & - & Tree \\
\hline 57 & Terminalia iverensis A. Chev & Black afara & Combretaceae & + & + & - & Tree (VU) \\
\hline
\end{tabular}




\section{Continued}

\begin{tabular}{|c|c|c|c|c|c|c|c|}
\hline 58 & $\begin{array}{c}\text { Terminalia superba Engl. \& } \\
\text { Diels }\end{array}$ & Terminalia or Afara & Combretaceae & + & + & - & Tree \\
\hline 59 & $\begin{array}{l}\text { Laguncularia racemosa (L.) } \\
\text { C.F. Gaertn. }\end{array}$ & White mangrove & Combretaceae & - & - & + & $\begin{array}{c}\text { Shrub } \\
\text { (Least concern) }\end{array}$ \\
\hline 60 & Commelina erecta $\mathrm{L}$. & White mouth dew flower & Commelinaceae & + & - & - & Herb \\
\hline 61 & Commelina benghalensis $\mathrm{L}$. & Wandering Jew & Commelinaceae & + & - & - & Herb \\
\hline 62 & Ipomoea aquatica Forssk & Ipomoea & Convulvulaceae & + & + & - & Trailing herb \\
\hline 63 & $\begin{array}{l}\text { Lepistemon owariense } \\
\text { (P. Beauv) Hallier F. }\end{array}$ & NA & Convulvulaceae & + & - & - & Twining perennial \\
\hline 64 & Ipomoea involucrata P. Beauv & NA & Convulvulaceae & + & - & - & Trailing herb \\
\hline 65 & Ipomoea batatas (L.) Lam & Sweet potato & Convulvulaceae & + & - & - & Trailing herb \\
\hline 66 & $\begin{array}{c}\text { Costus lucanusianus J. Braun } \\
\text { and K. Schum. }\end{array}$ & Bush cane & Costaceae & + & - & - & Shrub \\
\hline 67 & $\begin{array}{l}\text { Costus spectabilis Schum } \\
\text { (Fenzl) K. }\end{array}$ & Dwarf ginger lili & Costaceae & + & - & - & Herb \\
\hline 68 & Costus afer K. Gawl & Bush cane & Costaceae & + & - & - & Shrub \\
\hline 69 & $\begin{array}{l}\text { Lagenaria guineensis (G. Don) } \\
\text { C. Jeffrey }\end{array}$ & Cucurbit & Cucurbitaceae & + & + & - & Climbing Herb \\
\hline 70 & Zehnaria cinerea & NA & Cucurbitaceae & + & + & - & Climbing Herb \\
\hline 71 & Luffa aegyptiaca Mill. & Sponge gourd & Cucurbitaceae & + & - & - & Climbing Herb \\
\hline 72 & Momordica charantia L. & Bitter gourd & Cucurbitaceae & + & - & - & Climbing Herb \\
\hline 73 & $\begin{array}{l}\text { Coccinia barteriI (Hook. f) } \\
\text { Keay }\end{array}$ & NA & Cucurbitaceae & + & - & - & Climbing Herb \\
\hline 74 & Scleria verrucosa Wild & Bush knife & Cyperaceae & + & - & - & Herb \\
\hline 75 & Scleria naumanniana Boeck & Bush knife & Cyperaceae & + & - & - & Herb \\
\hline 76 & Bulbostylis sp & Sedge & Cyperaceae & + & - & - & Herb \\
\hline 77 & Kyllinga erecta Schumach & Sedge & Cyperaceae & + & - & - & Herb \\
\hline 78 & $\begin{array}{l}\text { Mariscus longibracteatus } \\
\text { Cherm }\end{array}$ & Sedge & Cyperaceae & - & + & - & Herb \\
\hline 79 & Mariscus flabelliformis Kunth & Sedge & Cyperaceae & + & - & - & Herb \\
\hline 80 & Kyllinga pumila Michx & Sedge & Cyperaceae & + & - & - & Herb \\
\hline 81 & Tetracera affinis Hutch & NA & Dilleniaceae & + & + & - & Scrambling shrub \\
\hline 82 & Dioscorea smilacifolia De Wild & Bush yam & Dioscoreaceae & + & + & - & Climber \\
\hline 83 & Dioscorea sansibarensis Pax & Bush yam & Dioscoreaceae & + & - & - & Climber \\
\hline 84 & Dracaena mannii Barker & Small-leaved dragon tree & Dracaenaceae & + & + & - & Tree \\
\hline 85 & $\begin{array}{l}\text { Dracaena arborea } \\
\text { (Willd.) Link }\end{array}$ & African dragon tree & Dracaenaceae & + & - & - & Tree \\
\hline 86 & Diospyros crassiflora Hiern & Ebony & Ebenaceae & + & + & - & Tree (Threatened) \\
\hline 87 & $\begin{array}{c}\text { Phyllanthus amarus Schum \& } \\
\text { Thonn }\end{array}$ & Stone breaker & Euphorbiaceae & + & + & - & Herb \\
\hline 88 & $\begin{array}{l}\text { Ricinodendron heudelotii } \\
\text { (Bill.) Pierre }\end{array}$ & Corkwood tree & Euphorbiaceae & + & + & - & Tree (VU) \\
\hline 89 & $\begin{array}{l}\text { Alchornea laxiflora } \text { (Benth) } \\
\text { Pax \& K. Hoffin }\end{array}$ & Christmas bush & Euphorbiaceae & + & + & - & Shrub \\
\hline
\end{tabular}




\section{Continued}

\begin{tabular}{|c|c|c|c|c|c|c|c|}
\hline 90 & $\begin{array}{l}\text { Manniophyton fulvum } \\
\text { Mull-Arg }\end{array}$ & NA & Euphorbiaceae & + & - & - & Shrub \\
\hline 91 & Bridella ferruginea Benth & NA & Euphorbiaceae & + & + & - & Tree \\
\hline 92 & $\begin{array}{c}\text { Alchornea cordifolia } \\
\text { (Schum \& Thonn) Mull.-Arg. }\end{array}$ & Christmas bush & Euphorbiaceae & + & + & - & Shrub \\
\hline 93 & Manihot esculenta Crantz & Cassava & Euphorbiaceae & + & - & - & Shrub \\
\hline 95 & Jatropha curcas Linn & Purge nut & Euphorbiaceae & + & - & - & Shrub \\
\hline 96 & $\begin{array}{c}\text { Antidesma vogelianum } \\
\text { Mull-Arg }\end{array}$ & NA & Euphorbiaceae & + & + & - & Tree \\
\hline 97 & Uapaca heudelotii Baill. & Uapaca & Euphorbiaceae & - & + & - & Tree \\
\hline 98 & $\begin{array}{c}\text { Milletia arboensis (Hook F.) } \\
\text { Bak }\end{array}$ & NA & Leguminosae & + & + & - & Tree \\
\hline 99 & $\begin{array}{l}\text { Berlinia gradiflora (Vahl) } \\
\text { Hutch \& Dalz }\end{array}$ & NA & Leguminosae & + & - & - & Tree \\
\hline 100 & Dialium guineense Wild & Velvet tamarind & Leguminosae & + & - & - & Tree \\
\hline 101 & $\begin{array}{c}\text { Desmodium scorpiurus (Sw) } \\
\text { Desv }\end{array}$ & NA & Leguminosae & + & + & - & Herb \\
\hline 102 & $\begin{array}{c}\text { Desmodium ramosissimum } \mathrm{G} . \\
\text { Don }\end{array}$ & NA & Leguminosae & + & + & - & Semi-woody Herb \\
\hline 103 & $\begin{array}{c}\text { Anthonotha macrophylla } \mathrm{P} . \\
\text { Beauv }\end{array}$ & NA & Leguminosae & + & + & - & Tree \\
\hline 104 & $\begin{array}{c}\text { Anthonotha obanensis (Bak T.) } \\
\text { Leonard }\end{array}$ & NA & Leguminosae & + & + & - & Tree \\
\hline 105 & Baphia nitida Lodd & Camwood & Leguminosae & + & + & - & Shrub \\
\hline 107 & Brachystegia eurycoma Harms & NA & Leguminosae & + & + & - & Tree \\
\hline 108 & Mimosa inuisa Mart ex. Colla & Sensitive plant & Leguminosae & + & + & - & Shrub \\
\hline 109 & $\begin{array}{c}\text { Tetrapleura tetraptera (Schum } \\
\text { \& Thonn. Taub) }\end{array}$ & NA & Leguminosae & + & + & - & Tree \\
\hline 110 & $\begin{array}{c}\text { Amphimas pterocarpoides } \\
\text { Hams. }\end{array}$ & Bokanga & Leguminosae & + & + & - & $\begin{array}{c}\text { Tree } \\
\text { (Not evaluated) }\end{array}$ \\
\hline 111 & $\begin{array}{c}\text { Dalbergia ecastaphyllum (L.) } \\
\text { Taub. }\end{array}$ & Coin vine & Leguminosae & - & - & + & $\begin{array}{c}\text { Shrub } \\
\text { (Not evaluated) }\end{array}$ \\
\hline 112 & $\begin{array}{c}\text { Albizia adiantifolia (Schum) W. } \\
\text { Wight }\end{array}$ & West African albizia & Leguminosae & + & + & - & Tree \\
\hline 113 & Mimosa pudica Linn & Sensitive plant & Leguminosae & + & - & - & Herb \\
\hline 114 & $\begin{array}{c}\text { Pentaclethra macrophylla } \\
\text { Benth }\end{array}$ & Oil bean & Leguminosae & + & + & - & Tree \\
\hline 115 & $\begin{array}{l}\text { Piptadeniastrum africanum } \\
\text { (Hook.f.) Brenam }\end{array}$ & $\begin{array}{l}\text { False sasswood or } \\
\text { African greenheart }\end{array}$ & Leguminosae & + & + & - & $\begin{array}{c}\text { Tree } \\
\text { (Not evaluated) }\end{array}$ \\
\hline 116 & $\begin{array}{c}\text { Pueraria phaseoloides (Roxb) } \\
\text { Benth }\end{array}$ & Tropical kudzu & Leguminosae & + & - & - & Climber \\
\hline 117 & Pterocarpus milbraedii Harms & NA & Leguminosae & + & - & - & Tree \\
\hline 118 & Pterocarpus santalinoides DC & NA & Leguminosae & + & - & - & $\begin{array}{c}\text { Tree } \\
\text { (Least concern) }\end{array}$ \\
\hline 119 & $\begin{array}{l}\text { Mucuna sloanei Fawc. } \\
\text { \& Rendle }\end{array}$ & Hamburger bean & Leguminosae & + & - & - & Climber \\
\hline
\end{tabular}




\section{Continued}

\begin{tabular}{|c|c|c|c|c|c|c|c|}
\hline 120 & Calopogonium mucunoides Desv & & Leguminosae & + & - & - & Climbing Herb \\
\hline 121 & Acacia kamerunensis Gandoger & NA & Leguminosae & + & - & - & Shrub \\
\hline 122 & $\begin{array}{c}\text { Harungana madagascarensis Poir } \\
\text { ex. Lam }\end{array}$ & Dragon's blood & Guttiferae & + & + & - & Tree \\
\hline 123 & Symphonia globulifera L.f. & Hog gum tree & Guttiferae & - & + & - & $\begin{array}{c}\text { Tree } \\
\text { (Data deficient) }\end{array}$ \\
\hline 124 & Pentadesma butyracea Sabine & Tallow tree & Guttiferae & + & + & - & $\begin{array}{c}\text { Tree } \\
\text { (Not evaluated) }\end{array}$ \\
\hline 125 & Icacina triacantha Oliv & NA & Icacinaceae & + & - & - & Herb \\
\hline 126 & $\begin{array}{c}\text { Irvingia gobonensis } \\
\text { (Aubry-Lecomte ex O’Rorke) } \\
\text { Baill }\end{array}$ & Bush mango or dikanut & Irvingiaceae & + & + & - & $\begin{array}{c}\text { Tree } \\
\text { (Near threatened) }\end{array}$ \\
\hline 127 & Klainedoxa gabonensis Pierre & Timber wood & Irvingiaceae & + & + & - & $\begin{array}{c}\text { Tree } \\
\text { (Not evaluated) }\end{array}$ \\
\hline 128 & Persea gratissima Mill & Avocado pear & Lauraceae & + & - & - & Tree (fruit) \\
\hline 129 & Anthocleista nobilis G. Don & Cabbage tree & Logoniaceae & + & - & - & Tree \\
\hline 130 & Spigelia anthelmia Linn & worm grain & Loganiaceae & + & - & - & Herb \\
\hline 131 & $\begin{array}{c}\text { Abelmoschus esculentus (Linn) } \\
\text { Moench }\end{array}$ & Okro & Malvaceae & + & - & - & Shrub \\
\hline 132 & Sida acuta Burm.f. & NA & Malvaceae & + & - & - & Herb \\
\hline 133 & Urena lobata L. & & Malvaceae & + & - & - & Shrub \\
\hline 134 & Marrattia fraxinea $\mathrm{Sm}$ & Pteridophyte & Marattiaceae & + & - & - & Herb \\
\hline 135 & $\begin{array}{l}\text { Marantochtoa leucantha } \\
\text { (K. Schum) Milne-Redh }\end{array}$ & Yoruba soft cane & Marantaceae & + & + & - & Herb \\
\hline 137 & Osbeckia tubulosa Smith & NA & Melastomataceae & - & + & - & Herb \\
\hline 138 & Khaya ivorensis A. Chev & Mahogany & Meliaceae & + & + & - & Tree (Threatened) \\
\hline 139 & $\begin{array}{c}\text { Entandrophragna cylindrium } \\
\text { Hams }\end{array}$ & Sapele mahogany & Meliaceae & + & + & - & Tree (VU) \\
\hline 140 & $\begin{array}{c}\text { Guarea cedrata (A. Chev.) } \\
\text { Pellegrin }\end{array}$ & Pink African cedar & Meliaceae & + & + & - & Tree (VU) \\
\hline 141 & Carapa procera DC & $\begin{array}{l}\text { Monkey cola or Crab } \\
\text { wood }\end{array}$ & Meliaceae & + & + & - & Tree \\
\hline 142 & Ficus sur Forssk & Bush fig & Moraceae & + & + & - & Tree \\
\hline 143 & Ficus mucoso Welw ex. Falcalh & Fig tree & Moraceae & + & - & - & Tree \\
\hline 144 & Ficus exasperata Vahl. & Sand paper & Moraceae & + & - & - & Shrub \\
\hline 145 & Milicia excelsa (Welw) C.C. Berg & Iroko tree & Moraceae & + & + & - & Tree (Threatened) \\
\hline 146 & Artocarpus altilis (Park) Fosberg & Bread fruit & Moraceae & + & + & - & Tree \\
\hline 147 & Morus mesozygia Stapf. & NA & Moraceae & + & + & - & Tree \\
\hline 148 & Treculia africana Decne & African bread fruit & Moraceae & + & + & - & Tree \\
\hline 149 & Musa sapientum L. & Banana & Musaceae & + & - & - & Tree \\
\hline 150 & Musa paradisiaca L. & Plantain & Musaceae & + & - & - & Tree \\
\hline 151 & Myristica fragrans & Nutmeg & Myristicaceae & + & + & - & Tree \\
\hline
\end{tabular}




\section{Continued}

\begin{tabular}{|c|c|c|c|c|c|c|c|}
\hline 152 & $\begin{array}{c}\text { Pycnanthus angolense (Welw.) } \\
\text { Warb. }\end{array}$ & Wild nutmeg & Myristicaceae & + & + & - & Tree \\
\hline 153 & Psidium guajava L. & Guava & Myrtaceae & + & - & - & Fruit Tree \\
\hline 154 & Eugenia owariensis L. & African rose apple & Myrtaceae & - & + & - & Tree \\
\hline 155 & Syzygium guineense (Willd.) DC. & Water berry & Myrtaceae & - & + & - & Tree \\
\hline 156 & Lophira alata Banks ex Gaertn. & Red ironwood & Ochnaceae & + & + & - & Tree (VU) \\
\hline 157 & Ludwigia abyssinica A. Richard & NA & Onagraceae & + & + & - & Herb \\
\hline 158 & Ludwigia decurrens Walter & Willow primrose & Onagraceae & + & + & - & Herb \\
\hline 159 & Bulbophyllum barbigerum Lindl & Epiphyte orchid & Orchidaceae & + & + & - & Herb \\
\hline 160 & Microdesmis puberula Hook F. & NA & Pandaceae & + & - & - & Shrub \\
\hline 161 & Pandanus candelabrum P. Beauv. & NA & Pandanaceae & - & + & - & Shrub \\
\hline 162 & Adenia lobata (Jacq) Engl. & NA & Passifloraceae & + & - & - & Herb \\
\hline 163 & Chloris pilosa Schumach & Finger grass & Poaceae & + & + & - & Herb \\
\hline 164 & $\begin{array}{c}\text { Pennisetum purpureum } \\
\text { Schumach }\end{array}$ & Elephant grass & Poaceae & + & - & - & Herb \\
\hline 165 & Eleusine indica (L) Gaertn & Wire grass & Poaceae & + & - & - & Herb \\
\hline 166 & Digitaria horizontallis & Grass & Poaceae & + & + & - & Herb \\
\hline 167 & $\begin{array}{c}\text { Axonopus compressus (SW.) P. } \\
\text { Beauv }\end{array}$ & Carpet grass & Poaceae & + & - & - & Herb \\
\hline 168 & Panicum laxum SW & Grass & Poaceae & + & + & - & Herb \\
\hline 169 & Panicum maximum Jacq & Guinea grass & Poaceae & + & - & - & Herb \\
\hline 170 & Bambusa vulgaris Schrad & Indian bamboo & Poaceae & + & + & - & Tree \\
\hline 171 & $\begin{array}{c}\text { Andropogon tectorum Schum \& } \\
\text { Thonn. }\end{array}$ & Giant blue stem & Poaceae & + & - & - & Herb \\
\hline 172 & $\begin{array}{l}\text { Sporobolus paniculatus (Trin) } \\
\text { (Trin) Dur. \& Schinz Dur }\end{array}$ & Grass & Poaceae & + & - & - & Herb \\
\hline 173 & Sporobolus pyramidalis P. Beauv & Rats tail grass & Poaceae & + & - & - & Herb \\
\hline 174 & $\begin{array}{c}\text { Chrysopogon aciculatus (Retz) } \\
\text { Trin }\end{array}$ & Love grass & Poaceae & + & - & - & Herb \\
\hline 175 & Eragrostis tenella (Linn) P. Beauv & Ross grass & Poaceae & + & - & - & Herb \\
\hline 176 & $\begin{array}{c}\text { Sorghum arundinaceum (Desv) } \\
\text { Stapf }\end{array}$ & Kamerun grass & Poaceae & - & + & - & Shrub \\
\hline 177 & $\begin{array}{c}\text { Acroceras zizanoides (Kunth) } \\
\text { Dandy }\end{array}$ & Grass & Poaceae & + & + & - & Herb \\
\hline 178 & Saccharum officinarum Linn & Sugar cane & Poaceae & - & + & - & Shrub \\
\hline 179 & Polygonium pulchrum Blume & NA & Polygonaceae & - & + & - & Herb \\
\hline 180 & $\begin{array}{c}\text { Eichhornia crassipes (Mart.) } \\
\text { Solms. }\end{array}$ & Water hyacinth & Pontederiaceae & - & + & - & Floating herb \\
\hline 181 & Talinum triangulare (Jacq) Wild & Waterleaf & Portulacaceae & + & - & - & Herb (vegetable) \\
\hline 182 & Portulaca oleracea L. & Common purslane & Portulacaceae & + & - & - & Herb (vegetable) \\
\hline 183 & $\begin{array}{c}\text { Rhizophora racemosa } \text { G.R.W } \\
\text { Meyer }\end{array}$ & Red mangrove & Rhizophoraceae & - & - & + & Tree \\
\hline 184 & Rhizophora mangle L. & Mangrove & Rhizophoraceae & - & - & + & Tree \\
\hline
\end{tabular}




\section{Continued}

\begin{tabular}{|c|c|c|c|c|c|c|c|}
\hline 185 & Rhizophora harrisonii Leechm & Mangrove & Rhizophoraceae & - & - & + & Tree \\
\hline 186 & Hallea ciliata Aubr \& Pellegr & Abura & Rubiaceae & + & + & - & Tree \\
\hline 187 & Oldenlandia corymbosa $\mathrm{L}$. & Flat top mille grained & Rubiaceae & - & + & - & Herb \\
\hline 188 & Ixora coccinea $\mathrm{L}$. & Jungle flame Ixora & Rubiaceae & + & - & - & Shrub (ornamental) \\
\hline 189 & Sabicea calycina Benth & NA & Rubiaceae & + & + & - & Shrub \\
\hline 190 & Mussaenda landolphioides Wernham & NA & Rubiaceae & - & + & - & Woody climber \\
\hline 191 & Mitracarpus scaber Zucc. & NA & Rubiaceae & + & - & - & Herb \\
\hline 192 & Nauclea latifolia $\mathrm{Sm}$. & NA & Rubiaceae & + & - & - & Tree \\
\hline 193 & Morinda lucida $\mathrm{L}$. & Brimstone tree & Rubiaceae & + & + & - & Tree \\
\hline 194 & Psychotria vogeliana Benth. & Wood cork & Rubiaceae & + & + & - & Shrub \\
\hline 195 & Rothmannia whitfieldii (Lindl.) Dandy & NA & Rubiaceae & + & + & - & Shrub \\
\hline 196 & Citrus sineensis (L.) Osbeck & Sweet orange & Rutaceae & + & - & - & Tree \\
\hline 197 & Paullinia pinnata $\mathrm{L}$. & Bread and cheese & Sapindaceae & + & + & - & Tree \\
\hline 198 & Scoparia dulcis L. & Sweet broom weed & Scrophulariaceae & + & - & - & Herb \\
\hline 199 & Selaginella myosurus (Sw.) Alston & Fern & Selaginellaceae & + & + & - & Herb \\
\hline 200 & Similax anceps Wild. & $\begin{array}{l}\text { West African } \\
\text { sarsaparilla }\end{array}$ & Smilacaceae & + & + & - & Climber \\
\hline 201 & Physalis angulata $\mathrm{L}$. & Angular winter cherry & Solanaceae & + & - & - & Herb \\
\hline 202 & Capsicum frutescens $\mathrm{L}$. & Red pepper & Solanaceae & + & - & - & Herb \\
\hline 203 & Solanum melongena $\mathrm{L}$. & Garden egg & Solanaceae & + & - & - & Herb \\
\hline 204 & Solanum torvum $\mathrm{Sw}$. & Turkey berry & Solanaceae & + & - & - & Shrub \\
\hline 205 & Capsicum annum $\mathrm{L}$. & Sweet red guinea pepper & Solonaceae & + & - & - & Herb \\
\hline 206 & Triplochiton scleroxylon K. Schum & Obeche & Sterculiaceae & + & + & - & Tree (Threatened) \\
\hline 207 & Sterculia tragacantha Lindl & African tragacanth & Sterculiaceae & + & + & - & Tree \\
\hline 208 & Chrysophyllum albidum G.Don & Star apple & Sterculiaceae & + & - & - & Tree \\
\hline 209 & Cola gigantea A. Chev. & Giant cola & Sterculiaceae & + & + & - & Tree (Threatened) \\
\hline 210 & Cola laurifolia Mast. & NA & Sterculiaceae & + & + & - & Tree (Threatened) \\
\hline 211 & Cola hispida Brenan \& Keay & NA & Sterculiaceae & + & - & - & Shrub \\
\hline 212 & Truimfetta esiophlebia Hook F. & African jute plant & Tiliaceae & + & + & - & Shrub \\
\hline 213 & Truimfetta cordifolia A. Rich & Bur weed & Tiliaceae & + & + & - & Shrub \\
\hline 214 & Glyphaea brevis (Spreng.) Monach & NA & Tiliaceae & + & + & - & Tree \\
\hline 215 & Clappertonia ficifolia (Willd.) Decne & Clappertonia & Tiliaceae & - & + & - & Shrub \\
\hline 216 & Duranta repens Linn & Golden dewberry & Verbenaceae & + & - & - & Shrub \\
\hline 217 & Gmelina arborea $\operatorname{Roxb}$ & Gmelina & Verbenaceae & + & - & - & Tree \\
\hline 218 & Vitex grandifolia Gurke & Black plum & Verbenaceae & + & + & - & Tree \\
\hline 219 & Aframonum melagueta $\mathrm{K}$. Schum. & Guinea pepper & Zingiberaceae & + & + & - & $\begin{array}{c}\text { Herb } \\
\text { (Not evaluated) }\end{array}$ \\
\hline \multicolumn{3}{|c|}{ Total number of species in each forest } & 192 & & 113 & 8 & \\
\hline
\end{tabular}

"Lowland = Moist Lowland Rainforest; ${ }^{* *}$ Freshwater $=$ both seasonal and permanent freshwater swamp forest; NA = not applicable i.e., no common name; $+=$ present; $-=$ not present. 
(Fabaceae) was represented by 24 species (10.96\%), Poaceae by 16 species (7.31\%), Euphorbiaceae by 11 species (5\%), Rubiaceae by 10 species (4.57\%), Apocynaceae and Arecaceae by 8 species $(3.65 \%$ each) Figure 3(A). While Asteraceae, Cyperaceae and Moraceae contributed seven species each, Combretaceae, Cucurbitaceae and Solanaceae each contributed five species of the identified 219 species. The family Sterculiaceae was represented by six species, while 3 out of the 4 recorded species of Meliaceae, which are commercial timber species, are threatened based on IUCN [30] Conservation Status. The remaining 25 families were represented by a species each (Table 1). The percentage contributions of the different families based on the habit of the plants is as follows: herbs 70 species (31.96\%), trees 92 species (42\%), climbers/liana 21 species $(9.59 \%)$ and shrub 36 species (16.44\%) as shown in Figure 3(B). The forest inventory revealed average of 22 stands of trees in each $10 \mathrm{~m}^{2}$ area of the lowland rainforest/freshwater swamp forest and average of 9 stands of mangrove trees in $10 \mathrm{~m}^{2}$ mangrove swamp forest. Based on this information, it is estimated that $8,778,000$ stands of lowland rainforest/freshwater swamp forest trees and 864,000 stands of mangroves were destroyed in the process of actualizing the construction of the pipelines.

\subsection{Status of the Wildlife of the Area}

Wildlife inventory (invertebrates not included) in the area revealed considerable diversity of wildlife species in 4 broad groups. This comprised 125 different fauna species from 64 families (Table 2-5). Mammals from 22 families (Table 2) accounted for 44 (35.2\% of total number of species) of the species described (Figure 4). 20 of these mammals including the Pan troglodytes-chimpanzee (EN), Cercopithecus erythrogaster-white throated

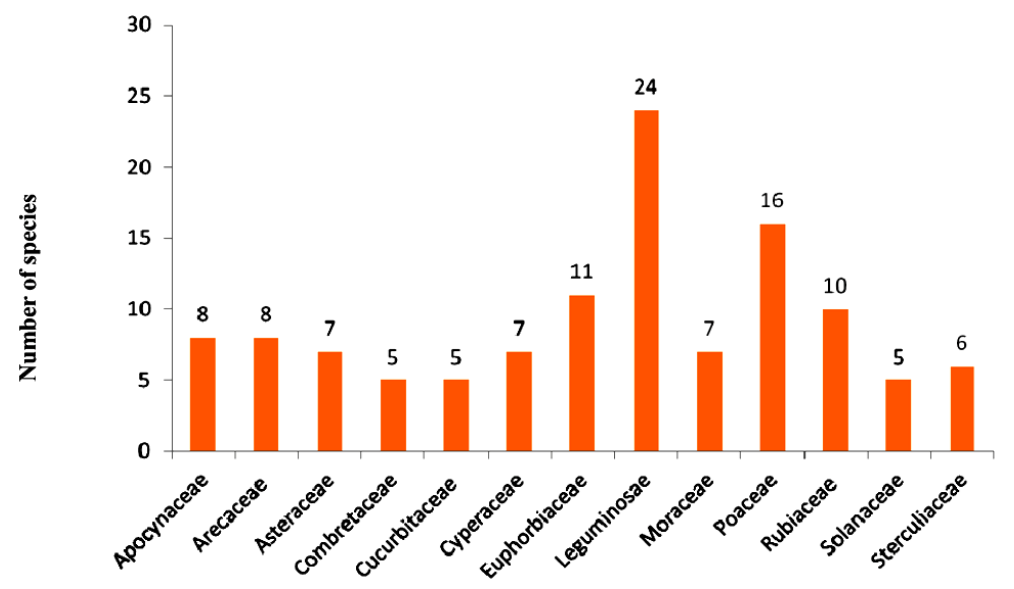

(a)

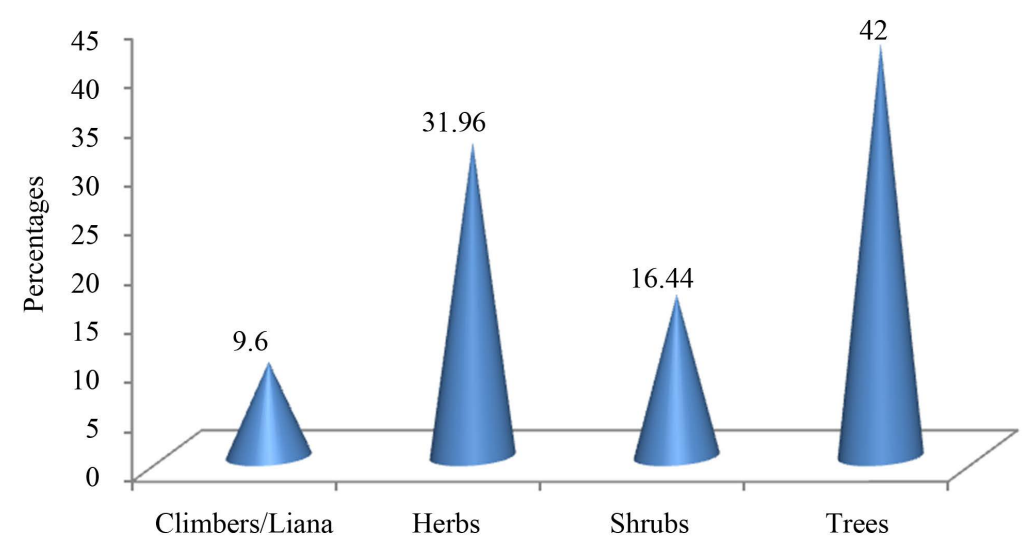

(b)

Figure 3. (a) Species composition of the most dominant plant families in the study areas; (b) Percentage contribution of different plant families (based on plant habit) to the vegetation of the study area. 


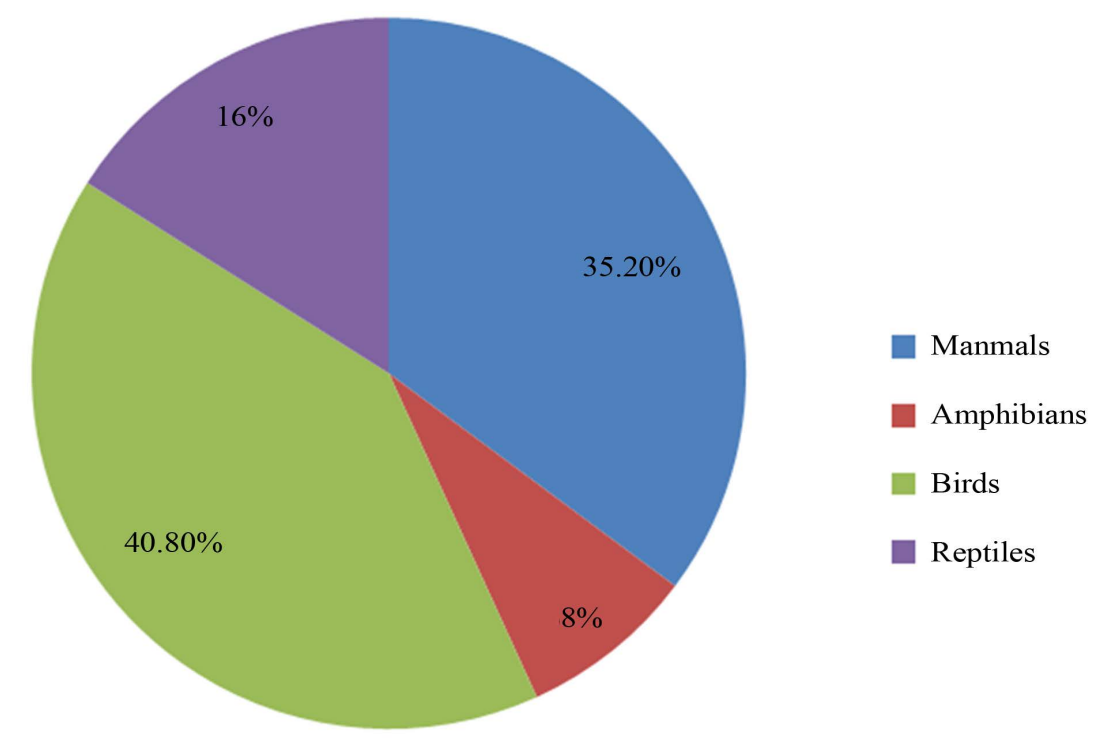

Figure 4. Percentage contribution of different wildlife groups in the study areas.

guenon (VU), Trichechus senegalensis-African civet (VU) and Panthera pardus-leopard (VU) are threatened or near threatened according to IUCN and Nigerian Conservation status. Other taxa represented in the records are 10 species of amphibians from 5 families (Table 3), 20 species of reptiles from 13 families (Table 4), and 51 species of birds from 24 families (Table 5). Birds accounted for $40.8 \%$ of the total faunal species in the area (Figure 4). 7 of the 51 species of birds, which includes grey parrot (VU), Anambra waxbill (VU) and hooded vulture (EN), are threatened. The goliath frog (an amphibian) and river turtle (reptile) are endangered, while the West African dwarf crocodile, another reptile, is vulnerable. These are threatened in line with IUCN and Nigerian Conservation status. Though the birds showed more species diversity, the amphibians were more abundant in population and Bufo regularis (the common African toad), was the most dominant amphibian. This species occurred in all the ecological zones. Three totems (Table 6), Kinixys erosa (serrated hinge-backed tortoise), $P$ thon sebae (Rock python), and Python regius (Royal python) were recorded.

\subsection{Critical Impacts on Biodiversity of the Area}

The impacts observed and recorded during the study include colossal destruction of plants and dislodgement of animals from their habitat (Figures 2(A)-(D), Figure 5(A) and Figure 5(B)), creation of multiple accesses into hitherto un-accessed forests resulting in increased logging/hunting of wildlife (Figures 2(A)-(D)). Within the lowland tropical rainforest, the RoW has converted to derived savanna (forest fragmentation) dominated by grasses, sedges and amphibians (Figure 2(B)). In the freshwater swamp areas, the same RoW was observed to be route for migration of invasive alien species like water hyacinth (Figure 5(C)). Other terrible impacts recorded are pipeline rupture leading to spillage into water bodies and farmland, loss of aquatic flora and fauna, fire incidents, destruction of forest biodiversity, and death of humans (Figures 5(A)-(F)), heightened flooding and erosion due to removal of vegetal cover and disruption of hydrologic patterns, and distortion of the ecotone zone of the freshwater swamp and mangrove forests.

\section{Discussion}

Results obtained from this study agree with the fact that diverse ecological zones translate to diversity in species; a scenario easily adducible for the Niger Delta from information presented in Tables 1-5. That the Niger Delta is replete with species of plants and animals including endemic species is well documented [1] [3] [6] [31] [32]. With 219 species of plants in 66 families, and 125 different fauna species from 64 families (excluding invertebrates) from the study area, this study lays credence and strongly supports previous reports that the Niger Delta is ecologically diverse in species.

Forests in the Niger Delta are productive and sensitive ecosystems dominated by rainforest and mangrove 

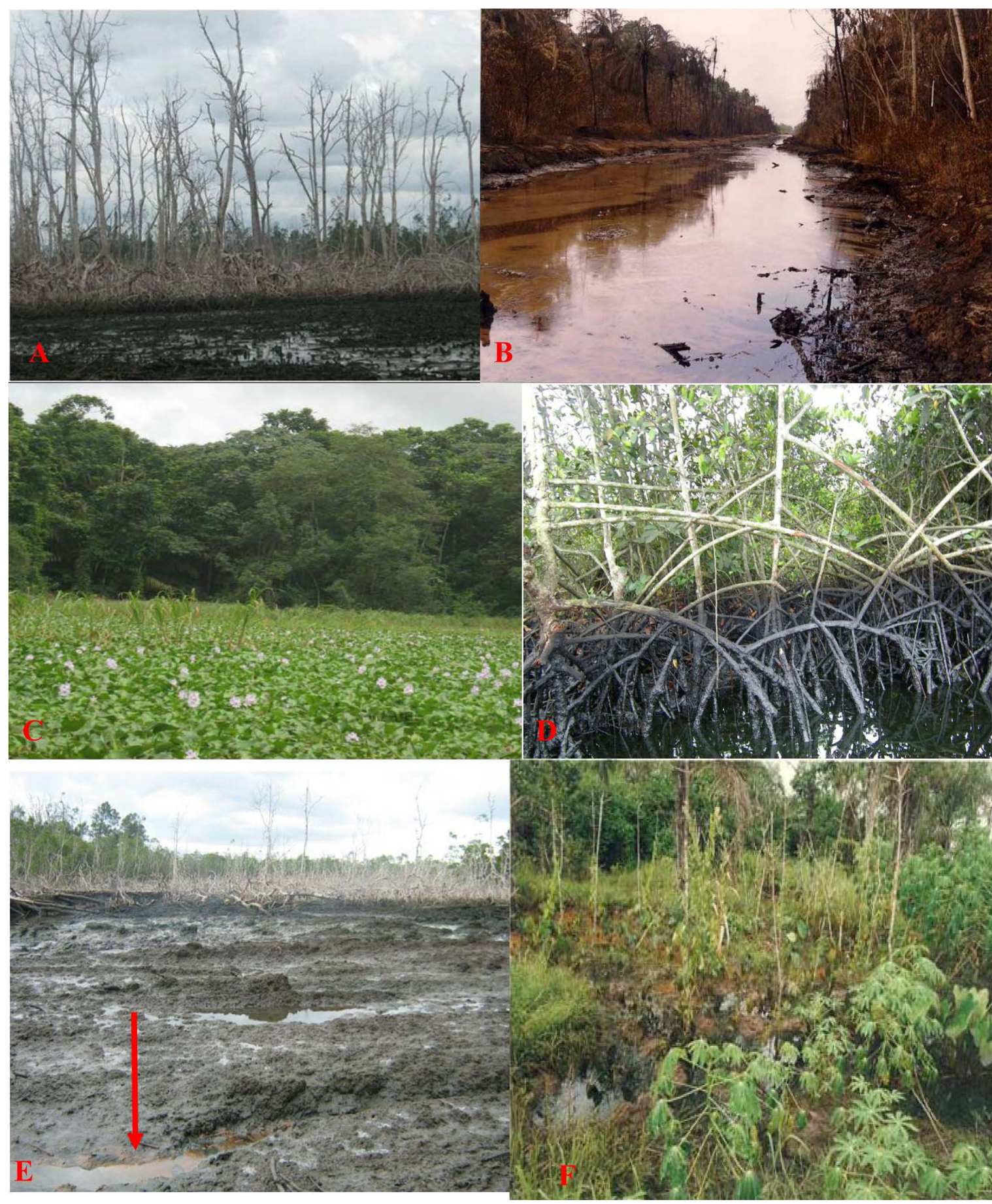

Figure 5. (A)-(F) Different impacts from the operational phase of the $120 \mathrm{~km}$ pipeline observed during the study: (A) Large scale destruction of mangroves resulting from fire incidence, which occurred after oil spillage from the pipeline; (B) Colossal kill of freshwater swamp forest and forest resources after an oil spill and fire incidence along the freshwater swamp section of the same pipeline. Notice crude oil streaming along the RoW. The RoW provides access for the streaming crude oil to freely move into water bodies, ponds, and farmlands along the course; (C) Water hyacinth (Eichhornia crassipes), an invasive alien species, along the RoW; (D) Stilt roots of Rhizophora sp. heavily covered with crude oil resulting from spillage along the mangrove section of the pipeline RoW; (E) Another section of 5A at low tide. Arrow points to pool of crude oil persisting in the mangrove "chikoko" soil two years after the spillage; (F) Farmland along the RoW, which was impacted by oil spill from the pipeline. Crops planted in the farm include cassava, yam and cocoyam. Communities along the drier section of the $120 \mathrm{~km}$ RoW are allowed to farm beside the RoW. 
Table 2. Fauna of the Study Area (Mammals).

\begin{tabular}{|c|c|c|c|c|}
\hline S/no & Family & Scientific Name & Common Name & IUCN Status \\
\hline 1 & Cercopithecidae & Cercopithecus nictitans (Linn, 1766) & Putty nose monkey & $\mathrm{LC}\left({ }^{*} \mathrm{EN}\right)$ \\
\hline 2 & Cercopithecidae & Cercopithecus erythrogaster (Gray, 1866) & White throated guenon & VU \\
\hline 3 & Cercopithecidae & Cercopithecus mona (Schreber, 1775) & Mona monkey & $\mathrm{LC}\left({ }^{*} \mathrm{NT}\right)$ \\
\hline 4 & Cercopithecidae & Procolobus verus (Van Beneden, 1838) & Olive colobus & NT \\
\hline 5 & Anomaluridae & Anomalurus beecrofti (Fraser, 1853) & Beecrott's flying squirrel & $\mathrm{LC}$ \\
\hline 6 & Anomaluridae & Anomalurus derbianus (Gray, 1842) & Derby's flying squirrel & $\mathrm{LC}$ \\
\hline 7 & Herpestidae & Atilax paludinosus (G.[Baron] Cuvier, 1829) & Marsh mongoose & $\mathrm{LC}$ \\
\hline 8 & Herpestidae & Herpestes naso (de Winton, 1901) & Long-nosed monogoose & $\mathrm{LC}$ \\
\hline 9 & Hystricidae & Atherurus africanus (Gray, 1842) & Brush-tailed porcupine & $\mathrm{LC}$ \\
\hline 10 & Bovidae & Tragelaphus spekii (Speke, 1863) & Sitatunga & $\mathrm{LC}\left({ }^{*} \mathrm{NT}\right)$ \\
\hline 11 & Bovidae & Tragelaphus scriptus (Pallas, 1766) & Bush buck & $\mathrm{LC}\left({ }^{*} \mathrm{NT}\right)$ \\
\hline 12 & Bovidae & Cephalophus maxwelli (H. Smith, 1827) & Maxwell's duiker & $\mathrm{LC}\left({ }^{*} \mathrm{NT}\right)$ \\
\hline 13 & Bovidae & Cephalophus silvicultor (Afzelius, 1815) & Yellow-backed duiker & $\mathrm{LC}\left({ }^{*} \mathrm{NT}\right)$ \\
\hline 14 & Bovidae & Cephalophus ogilbyi (Waterhouse, 1838) & Ogilby’s duiker & $\mathrm{LC}\left({ }^{*} \mathrm{NT}\right)$ \\
\hline 15 & Bovidae & Syncerus caffer (Sparrman, 1779) & African buffalo & $\mathrm{LC}\left({ }^{*} \mathrm{EN}\right)$ \\
\hline 16 & Nesomyidae & Cricetomys emini (Wroughton, 1910) & Emini’s giant-rat & $\mathrm{LC}$ \\
\hline 17 & Galagidae & Galagoides demidovii (G. Fischer, 1806) & Dwarf galago & $\mathrm{LC}$ \\
\hline 18 & Muridae & Lemniscomys striatus (Linnaeus, 1758) & Spotted grass-mouse & $\mathrm{LC}$ \\
\hline 20 & Muridae & Mus minutoides (Smith, 1834) & Pigmy mouse & $\mathrm{LC}$ \\
\hline 21 & Muridae & Rattus rattus (Linnaeus, 1758) & Black house rat & $\mathrm{LC}$ \\
\hline 22 & Muridae & Mastomys natalensis (Smith, 1834) & Multimammate mouse & $\mathrm{LC}$ \\
\hline 23 & Lorisidae & Perodicticus potto (Müller, 1766) & Bosman's potto & $\mathrm{LC}\left({ }^{*} \mathrm{NT}\right)$ \\
\hline 24 & Lorisidae & Arctocebus calabarensis (J.A. Smith, 1860) & Angwantibo & $\mathrm{LC}$ \\
\hline 25 & Manidae & Manis tetradactyla (Linn, 1766) & Long-tailed pangolin & $\mathrm{LC}\left({ }^{*} \mathrm{EN}\right)$ \\
\hline 26 & Manidae & Manis tricuspis (Rafinesque, 1821) & Tree pangolin & NT \\
\hline 27 & Muscardinidae & Graphiurus hueti Jentink, 1888 & Common African dormouse & $\mathrm{LC}$ \\
\hline 28 & Mustelidae & Aonyx capensis (Schinz, 1821) & Carpe clawless otter & $\mathrm{LC}\left({ }^{*} \mathrm{NT}\right)$ \\
\hline 29 & Mustelidae & Lutra maculicollis (Lichtenstein, 1835) & Spotted-necked otter & $\mathrm{LC}\left({ }^{*} \mathrm{VU}\right)$ \\
\hline 30 & Soricidae & Crocidura nigeriae (Dollman, 1915) & Nigerian musk shrew & $\mathrm{LC}$ \\
\hline 31 & Suciuridae & Protoxerus stangeri (Waterhouse, 1842) & Giant forest-squirrel & $\mathrm{LC}$ \\
\hline 32 & Suciuridae & Heliosciurus rufobrachium (Waterhouse, 1842) & Red-legged sun squirrel & $\mathrm{LC}$ \\
\hline 33 & Suciuridae & Funisciurus anerythrus (Thomas, 1890) & Red-less tree-squirrel & $\mathrm{LC}$ \\
\hline 34 & Suidae & Potamochoerus porcus (Linnaeus, 1758) & Red river-hog & $\mathrm{LC}\left({ }^{*} \mathrm{VU}\right)$ \\
\hline 35 & Thryonomidae & Thryonomys swinderianus (Temminck, 1827) & Greater cane rate & $\mathrm{LC}$ \\
\hline
\end{tabular}




\begin{tabular}{|c|c|c|c|c|}
\hline \multicolumn{5}{|c|}{ Continued } \\
\hline 36 & Tragulidae & Hyemoschus aquaticus (Ogilby, 1841) & Water chevrotain & $\mathrm{LC}\left({ }^{*} \mathrm{DD}\right)$ \\
\hline 37 & Trichechidae & Trichechus senegalensis (Link, 1795) & African civet & VU \\
\hline 38 & Vevirridae & Viverra civetta (Schreber, 1776) & Two-spot palm civet & $\mathrm{LC}$ \\
\hline 39 & Vevirridae & Nandinia binotata (Gray, 1830) & Two-spot palm civet & $\mathrm{LC}$ \\
\hline 40 & Vevirridae & Genetta cristata (Hayman in Sanborn, 1940) & Crested genet & VU \\
\hline 41 & Vevirridae & Genetta pardina (Geoffrey Saint-Hilaire, 1832) & Forest genet & $\mathrm{LC}$ \\
\hline 42 & Pteropodidae & Hypsignathus monstrosus (H. Allen, 1861) & Hammer- headed fruit bat & $\mathrm{LC}$ \\
\hline 43 & Felidae & Panthera pardus (Linnaeus, 1758) & Leopard & NT ( $\left.{ }^{*} \mathrm{VU}\right)$ \\
\hline 44 & Hominidae & Pan troglodytes (Blumenbach, 1799) & Chimpanzee & EN \\
\hline
\end{tabular}

Table 3. Fauna of the Study Area (Amphibians).

\begin{tabular}{ccccc}
\hline S/no & Family & Scientific Name & Common Name & IUCN Status \\
\hline 1 & Bufonidae & Bufo regularis (Reuss, 1833) & African Common Toad & LC \\
2 & Bufonidae & Bufo maculates (Hallowell, 1855) & Toad & LC \\
3 & Bufonidae & Bufo gracillipes (Boulenger, 1899) & Toad & LC \\
4 & Ranidae & Rana temporaria (Linnaeus, 1758) & Common frog & LC \\
5 & Ranidae & Hylarana albolabris (Hallowell, 1856) & Frog & LC \\
6 & Ranidae & Rana clamitans (Latreille, 1801) & Green frog & LC \\
7 & Ranidae & Ptychadenamascareniensis (Dumeril\&Bibron, 1841) & True frog & LC \\
8 & Pipidae & Xenopus tropicalis (Gray, 1864) & Clawed toe frog & LC \\
9 & Hylidae & Hyla arborea (Linnaeus, 1758) & Tree frog & LC \\
10 & Conrauidae & Conraua goliath (Boulenger, 1906) & Goliath frog & EN \\
\hline
\end{tabular}

trees [3]. They are of considerable ecological importance not only because of their use as spawning and feeding grounds for many varieties of fish and shrimps but also of economic importance because forests are sources of timber, poles, fire wood, palm oil and other local food crops and medicinals. The forests support diverse wildlife species including totems as demonstrated by the results of this study. However, oil and gas exploration and production activities have over the years impacted negatively (directly and indirectly) on the biological diversity of this region resulting in increasing rate of threat to species and extinction of others (Tables 1-5). Opening up of landlocked rich forest ecosystems during such activities as pipeline construction encourage unsustainable forest exploitation, environmental degradation and biodiversity loss in the area. These activities accentuate flooding and flooding impacts, and the operational consequences of these pipelines, which include vandalization and oil spillage, exacerbate greenhouse gas emission and global warming. Oil spillage from oil and gas facilities and their impacts on biodiversity, soil, water, agricultural lands and humans in the Niger Delta is widely reported [33]-[38], and are confirmed by this study. That the construction and laying of flowlines/pipelines leading to the clearing of vegetation and excavation of earth has caused destruction and loss of forests and biodiversity thereby changing the landscape of the Niger Delta is not questionable. With an estimated whooping 8,778,000 stands of lowland rainforest/freshwater swamp forest trees and 864,000 stands of mangroves that were destroyed with construction and laying of pipeline along the studied $165 \mathrm{~km}$ stretch, the impact of the over $2000 \mathrm{~km}$ network of oil and gas pipelines in the Niger Delta can be imagined. This makes forest trees and plants the most affected 
Table 4. Fauna of the Study Area (Reptiles).

\begin{tabular}{|c|c|c|c|c|}
\hline S/no & Family & Scientific Name & Common Name & IUCN Status \\
\hline 1 & Agamidae & Agama agama (Loveridge, 1923) & Agama lizard (Rainbow lizard) & LC \\
\hline 2 & Chamaeleonidae & Chamaeleo gracilis (Hallowell, 1857) & Graceful chameleon & $\mathrm{LC}$ \\
\hline 3 & Colubridae & Gastropyxis smaragdina (Schlegel, 1837) & Emerald snake & Not evaluated \\
\hline 4 & Colubridae & Grayia smythii (Leach, 1818) & Smith's Water Snake & Not evaluated \\
\hline 5 & Colubridae & Bothropthalmus lineatus (Schlegel, 1856) & Red lined snake & Not evaluated \\
\hline 6 & Crocodylidae & Osteolaemus tetraspis (Cope, 1861) & West African dwarf crocodile & VU \\
\hline 7 & Crocodylidae & Crocodylus niloticus (Laurenti, 1768) & Nile crocodile & $\mathrm{LC}$ \\
\hline 8 & Elapidae & Dendroaspis viridis (Hallowell, 1844) & Green mamba & $\mathrm{LC}$ \\
\hline 9 & Elapidae & Naja nigricollis (Reinhardt, 1843) & Black-necked cobra & Not evaluated \\
\hline 10 & Elapidae & Naja melanoleuca & Black spiting cobra & Not evaluated \\
\hline 11 & Elapidae & Dispholidus typus (A. Smith, 1829) & Boomslang & Not evaluated \\
\hline 12 & Scincidae & Mochlus fernandi (Burton, 1836) & Forest skink & Not evaluated \\
\hline 13 & Pelomeduscidae & Pelusios niger (Lacépède, 1788) & West African black forest turtle & $\mathrm{LC}$ \\
\hline 14 & Pythonidae & Python sebae (Gmelin, 1788) & African Rock python & Not evaluated \\
\hline 15 & Pythonidae & Python reguis (Shaw, 1802) & Royal python & $\mathrm{LC}$ \\
\hline 16 & Boidae & Calabaria reinhardtii (Stimson, 1969) & Calabar ground python & $\mathrm{LC}$ \\
\hline 17 & Testudinidae & Kinixys erosa (Schweigger, 1812) & Serrated hinge-backed tortoise & $\mathrm{DD}$ \\
\hline 18 & Pelomedusidae & Pelomedusa subrufa (Lacépède 1788) & Forest turtle & $\mathrm{LC}$ \\
\hline 19 & Trionychidae & Trionyx triunguis (Forsskål, 1775) & River turtle & $\mathrm{EN}$ \\
\hline 20 & Viperidae & Bitis gabonica (Duméril, Bibron and Duméril, 1854) & Gabon viper & Not evaluated \\
\hline
\end{tabular}

biodiversity of the area. It must be stated that the removal of these trees has direct negative consequences on the wildlife, which co-habit with them; hence the number of fauna threatened with extinction in Tables 2-5. These fauna were automatically dislodged and or destroyed.

At present only less than $4 \%$ of Nigeria's rainforest cover is left. More frightening is the fact that the loss is still continuing at the rate of 3.5\% annually [39]. This implies colossal loss of biodiversity. This study has identified oil and gas pipeline construction as major culprit in moist lowland/freshwater swamp and mangrove swamp forests destruction in the Niger Delta.

\section{Conclusion}

It is noteworthy that in Nigeria, oil and gas exploration and production is sine qua non for government earnings and therefore tied to national development. However, it has caused unimaginable destruction to biodiversity and humans, and affected means of rural livelihood. Every caution must therefore be taken to apply best practice in the business of oil and gas exploration and production as applicable in other countries. Pragmatic efforts and policies geared towards reducing land-take for pipeline construction by using existing RoW for new flowlines/pipelines must be pursued and encouraged by the appropriate government arms. The RoW width of $30 \mathrm{~m}$ utilized for construction of most pipelines should be reduced to between $15 \mathrm{~m}$ and $20 \mathrm{~m}$ as one of the mitigations for impacts associated with vegetation clearing and biodiversity loss. There is also need to enforce restrain on construction of pipelines across identified local biodiversity hotspots in the region. At the moment, surveillance against pipeline rupture, failure and vandalism seems to be reactionary as against realistic pragmatism. Government must therefore liaise with concerned companies to evolve functional RoW surveillance methods, which 
Table 5. Fauna of the Study Area (Birds).

\begin{tabular}{|c|c|c|c|c|}
\hline S/no & Family & Scientific Name & Common Name & IUCN Status \\
\hline 1 & Accipitridae & Gypohierax angolensis (Gmelin, 1788) & Palm-nut vulture & LR ("NT) \\
\hline 2 & Accipitridae & Necrosyrtes monachus (Temminck, 1823) & Hooded vulture & EN \\
\hline 3 & Accipitridae & Polyboroides radiates (Scopoli, 1786) & Harrier hawk & $\mathrm{LC}$ \\
\hline 4 & Accipitridae & Haliaeetus vocifer (Daudin, 1800) & West African river eagle & $\mathrm{LC}$ \\
\hline 5 & Accipitridae & Stephanoaetus coronatus (Linnaeus, 1766) & Crown hawk eagle & NT \\
\hline 6 & Accipitridae & Milvus migrans (Boddaert, 1783) & Black kite & LR ( $\left({ }^{*} \mathrm{NT}\right)$ \\
\hline 7 & Accipitridae & Kaupifalco monogrammicus (Temminck, 1824) & Lizard buzzard & LR ("NT) \\
\hline 8 & Alcedinidae & Halcyon senegalensis (Linn, 1766) & Senegal kingfisher & $\mathrm{LC}$ \\
\hline 9 & Alcedinidae & Ceryle rudis (Linn, 1758) & Pied kingfisher & $\mathrm{LC}$ \\
\hline 10 & Alcedinidae & Ceyx pictus (Boddaert, 1783) & Pigmy kingfisher & $\mathrm{LC}$ \\
\hline 11 & Alcedinidae & Alcedo leucogaster (Fraser, 1843) & White-bellied kingfisher & $\mathrm{LC}$ \\
\hline 12 & Anatidae & Dendrocygna viduata (Linn, 1766) & White-faced tree duck & $\mathrm{LC}$ \\
\hline 13 & Ardeidae & Casmerodius albus (Linn, 1758) & Great white egret & $\mathrm{LC}$ \\
\hline 14 & Ardeidae & Egretta garzetta (Linn, 1766) & Little egret & $\mathrm{LC}$ \\
\hline 15 & Ardeidae & Ardea cinerea (Linn, 1758) & Grey heron & $\mathrm{LC}$ \\
\hline 16 & Ardeidae & Ardeola ralloides (Scopoli, 1769) & Squacco heron & LC \\
\hline 17 & Bucerotidae & Bycanistes fistulator (Cassin, 1852) & Piping hornbill & $\mathrm{LC}$ \\
\hline 18 & Bucerotidae & Tockus fasciatus (Shaw, 1811) & Black-and-white tail hornbill & $\mathrm{LC}$ \\
\hline 19 & Bucerotidae & Tropicranus albocristatus (Cassin, 1848) & White-crested hornbill & $\mathrm{LC}$ \\
\hline 20 & Bucerotidae & Ceratogymna atrata (Temminck, 1835) & Black-casqued hornbill & $\mathrm{LC}$ \\
\hline 21 & Columbidae & Treron australis (Linnaeus, 1771) & Green fruit pigeon & $\mathrm{LC}$ \\
\hline 22 & Columbidae & Treron waalia (Meyer, 1793) & Yellow-bellied fruit pigeon & $\mathrm{LC}$ \\
\hline 23 & Columbidae & Stigmatopelia senegalensis (Linn, 1766) & Laughing dove & $\mathrm{LC}$ \\
\hline 24 & Corvidae & Corvus albus (Müller, 1776) & Pied crow & $\mathrm{LC}$ \\
\hline 25 & Cuculidae & Centropus senegalensis (Linn, 1766) & Senegal coucal & $\mathrm{LC}$ \\
\hline 26 & Cuculidae & Centropus leucogaster (Leach, 1814) & Black-throated coucal & LC \\
\hline 27 & Estrilididae & Estrilda poliopareia (Reichenow, 1902) & Anambra waxbill & VU \\
\hline 28 & Hirundinidae & Hirundo smithii (Leach, 1818) & Wire-tailed swallow & $\mathrm{LC}$ \\
\hline 29 & Hirundinidae & Hirundo rustica (Linnaeus, 1758) & European Swallow & $\mathrm{LC}$ \\
\hline 30 & Jacanidae & Actophilornis africana (Gmelin, 1789) & Lilly-trotter & $\mathrm{LC}$ \\
\hline 31 & Meropidae & Merops albicollis (Vieillot, 1817) & White-throated bee-eater & LC \\
\hline 32 & Meropidae & Merops hirundineus (Lichtenstein, 1793) & Swallow-tailed bee-eater & $\mathrm{LC}$ \\
\hline 33 & Motacillidae & Motacilla flava (Linn, 1758) & Yellow wagtail & LC \\
\hline 34 & Musophygidae & Corythaeola cristata (Vieillot, 1816) & Great Blue Turaco & $\mathrm{LC}$ \\
\hline 35 & Musophygidae & Tauraco persa (Linn, 1758) & Guinea Turaco & $\mathrm{LC}$ \\
\hline
\end{tabular}




\section{Continued}

\begin{tabular}{|c|c|c|c|c|}
\hline 36 & Nectariniidae & Nectarinia venusta (Shaw \& Nodder, 1799) & Yellow-bellied sun-bird & LC \\
\hline 37 & Nectariniidae & Nectarinia olivacea (Smith, 1840) & Olive sun-bird & LC \\
\hline 39 & Oriolidae & Oriolus brachyrhynchus (Swainson, 1837) & Black-headed oriole & LC \\
\hline 40 & Phasianidae & Guttera edouardi (Hartlaub 1867) & Crested guinea fowl & $\mathrm{LC}$ \\
\hline 41 & Passeridae & Passer griseus (Vieillot, 1817) & Grey-headed sparrow & $\mathrm{LC}$ \\
\hline 42 & Ploceidae & Ploceus aurantius (Viellot, 1805) & Orange weaver & $\mathrm{LC}$ \\
\hline 43 & Ploceidae & Ploceus cucullatus (Müller, 1776) & Village weaver & $\mathrm{LC}$ \\
\hline 44 & Ploceidae & Ploceus melanocephalus (Linn, 1758) & Black-headed weaver & $\mathrm{LC}$ \\
\hline 46 & Ploceidae & Malimbus scutatus (Cassin, 1849) & Red-vented malimbe & $\mathrm{LC}$ \\
\hline 47 & Psittacidae & Psittacus erithacus (Linnaeus, 1758) & Grey parrot & VU \\
\hline 48 & Pycnonotidae & Pycnonotus barbatus (Desfontaine, 1789) & Common garden bulbul & $\mathrm{LC}$ \\
\hline 49 & Scolopacidae & Tringa hypoleucos (Linns, 1758) & Common sand piper & $\mathrm{LC}$ \\
\hline 50 & Turdidae & Cercotrichas galactotes (Temminck, 1820) & Rufous-tailed Scrub Robin & $\mathrm{LC}$ \\
\hline 51 & Viduidae & Vidua macroura (Pallas, 1764) & Pin-tailed whydah & $\mathrm{LC}$ \\
\hline
\end{tabular}

Table 6. Wildlife Species Considered totem, or associated with taboos in the study area.

\begin{tabular}{ccc}
\hline Family & Scientific Name & Common Name \\
\hline Varanidae & Kinixys erosa & Serrated hinge-backed tortoise \\
Pythonidae & Python sebae & Rock python \\
Pythonidae & Python regius & Royal python \\
\hline
\end{tabular}

$\mathrm{EN}=$ Endangered, $\mathrm{VU}=$ Vulnerable, $\mathrm{LR} / \mathrm{NT}=$ Lower Risk/Near Threatened, LR/CD $=$ Lower Risk/Conservation-Dependent, LC $=$ Least Concern, DD $=$ Data Deficient.

will completely eliminate pipeline vandalisation and its attendant consequences. This should as much as possible include robust education and awareness campaigns on the impacts of pipeline vandalization on biodiversity and humans. Where possible, the use of RoW for farming should be encouraged with extreme caution and supervision.

\section{References}

[1] Environmental Resources Managers Limited (ERML) (1997) Environmental and Social Characteristics of the Niger Delta. Phase 1: Vol. 1. A Report Submitted by Environmental Resources Managers Limited to the Niger Delta Environmental Survey (NDES), Lagos.

[2] Ibe, A.C. and Awosika, L.F. (1989) National Assessment of the Effects of Sea Level Rise on the Nigerian Coastal Zone. Proceedings of the International Conference on Global Climate Change at the Center for Climate Change, Maryland, 9-23 September 1989, 164-199.

[3] World Bank (1995) Defining an Environmental Development Strategy for the Niger Delta. Vol. 1, Report No. 14266, 1-149.

[4] Moffat, D. and Linden, O. (1995) Perception and Reality: Assessing Sustainable Development in the Niger River Delta. Ambio, 24, 527-538.

[5] Ohimain, E.I. (2003) Environmental Impacts of Oil Mining Activities in the Niger Delta Mangrove Ecosystem. In: Armstrong, D., de Villiers, A.B., Kleinmann, R.L.P., McCarthy, T.S. and Norton, P.J., Eds., Proceedings of the 8th In- 
ternational Mine Water Association (IMWA) Conference, International Mine Water Association (IMWA), Sandton, 2003, 503-517.

[6] Agbagwa, I.O. (2008) Impact of the Construction of Access Roads to Oil Well Locations and Flow Stations on the Biodiversity of Some Niger Delta Floodplains. Journal of Applied Science Research, 4, 1876-1884.

[7] Agbagwa, I.O. and Akpokodje, E.G. (2010) Canalization and Oil Pipeline Right-of-Way Construction: A Source of Saltwater Intrusion and Freshwater Swamp Forest Biodiversity Depletion in the Niger Delta. Scientia Africana, 9, 221 231.

[8] Mmom, P.C. and Arokoyu, S.B. (2010) Mangrove Forest Depletion, Biodiversity Loss and Traditional Resources Management Practices in the Niger Delta, Nigeria. Research Journal of Applied Sciences, Engineering and Technology, 2, 28-34.

[9] Spalding, M., Blasco, F. and Field, C. (1997) World Mangrove Ecosystem Atlas. The International Society for Mangrove Ecosystem (ISME), Japan.

[10] US Energy Information Administration, EIA (2011) Nigeria: Country Analysis Briefs. http://www.opec.org/opec_web/en/about_us/167.htm.

[11] Ashton-Jones, N. (1998) The Human Ecosystems of the Niger Delta. An ERA Handbook, Environmental Right Action (ERA), Benin City, 1-224.

[12] Okonta, I. and Douglas, O. (2001) Where Vultures Feast-Shell, Human Rights and Oil in the Niger Delta. Sierra Club Books, San Francisco, 1-286.

[13] Ohimain, E.I. (2004) Environmental Impacts of Dredging in the Niger Delta; Options for Sediment Relocation That Will Mitigate Acidification and Enhance Natural Mangrove Restoration. Terra et Aqua, 97, 9-19.

[14] Ohimain, E.O., Imoobe, T.O.T. and Benka-Coker, M.O. (2002) Impacts of Dredging on Zooplankton Communities of Warri River, Niger Delta. African Journal of Environmental Pollution and Health, 1, 37-45.

[15] Ohimain, E.I., Andriesse, W. and Van Mensvoort, M.E.F. (2004) Environmental Impacts of Abandoned Dredge Soils and Sediments: Available Options for Their Handling, Restoration and Rehabilitation. Journal of Soils and Sediments, 4, 59-65. http://dx.doi.org/10.1007/BF02990830

[16] Ohimain, E.O., Benka-Coker, M.O. and Imoobe, T.O.T. (2005) The Impacts of Dredging on Macrobenthic Invertebrates in a Tributary of the Warri River, Niger Delta. African Journal of Aquatic Sciences, 30, 49-53. http://dx.doi.org/10.2989/16085910509503834

[17] Osuji, L.C., Ndukwu, B.C., Obute, G.C. and Agbagwa, I. (2006) Impact of Four-Dimensional Seismic and Production Activities on the Mangrove Systems of the Niger Delta, Nigeria. Chemistry and Ecology, 22, 415-424. http://dx.doi.org/10.1080/02757540600917559

[18] Agbagwa, I.O. and Ekeke, C. (2011) Structure and Phytodiversity of Freshwater Swamp Forest in Oil-Rich Bonny, Rivers State, Nigeria. Research Journal of Forestry, 5, 66-77. http://dx.doi.org/10.3923/rjf.2011.66.77

[19] Richards, P.W. (1976) The Tropical Rainforest: An Ecological Study. Cambridge University Press, Cambridge.

[20] Hutchinson, J. and Dalziel, J.M. (1954) Flora of West Tropical Africa, Vol. 1, Part 1. Crown Agents for Oversea Governments and Administrations, London, 32-294.

[21] Hutchinson, J. and Dalziel, J.M. (1958) Flora of West Tropical Africa, Vol. 1, Part 2. Crown Agents for Oversea Governments and Administrations, London, 298-828.

[22] Hutchinson, J. and Dalziel, J.M. (1963) Flora of West Tropical Africa, Vol. 3, Part 1. Crown Agents for Oversea Governments and Administrations, London, 1-276.

[23] Keay, R.W.J. (1990) Trees of Nigeria. Clarendon Press, Oxford.

[24] Walker, E.P., Warnick, F. and Hamlet, S.E. (1968) Mammals of the World. Johns Hopkins Press, Baltimore, 2 Volumes, $1500 \mathrm{p}$.

[25] Elgood, J.H. (1960) Birds of the West African Town and Garden. West African Nature Handbooks Series, Longmans, London, 1-66.

[26] Booth, A.H. (1960) Small Mammals of West Africa. West African Nature Handbooks Series. Longmans, London, 168.

[27] Happold, D.C.D. (1987) The Mammals of Nigeria. Oxford University Press, New York, 1-402.

[28] Branch, B. (1998) Field Guide to the Snakes and Other Reptiles of Southern Africa. Ralph Curtis Pub., Florida, 328.

[29] Dasmann, D.F. (1964) Wildlife Biology and Conservation. John Wiley \& Sons Inc., New York, 1964, viii+231.

[30] World Conservation Union, IUCN (2006) IUCN Red List of Threatened Species. http://www.iucnredlist.org

[31] Powell, C.B. (1993) Sites and Species of Conservation Interest in the Central Axis of the Niger Delta (Yenagoa, Sag- 
bama, Ekeremor and Southern Ijo Local Government Areas). A Report of Recommendations to the Natural Resources Conservation Council (NARESCON), pp. 1-105 plus Maps and Appendices.

[32] Blench, R. (2007) Mammals of the Niger Delta, Nigeria. Cambridge, 1-64. http://www.rogerblench.info/RBOP.htm

[33] Odeyemi, O. and Ogunseitan, O.A. (1985) Petroleum Industry and Its Pollution Potential in Nigeria. Oil and Petroleum Pollution, 2, 223-229. http://dx.doi.org/10.1016/S0143-7127(85)90218-2

[34] Umechuruba, C.I. (2005) Health Impact Assessment of Mangrove Vegetation in an Oil Spilled Site at the Bodo West Field in Rivers State. Nigeria Journal of Applied Science and Environmental Management, 9, 69-73.

[35] Ndubuisi, A.L. and Asia, I.O. (2007) Environmental Pollution in Oil Producing Areas of the Niger Delta Basin, Nigeria: Empirical Assessment of Trends and People's Perception. Environmental Research Journal, 1, 18-26.

[36] Eregha, P.B. and Irughe, I.R. (2009) Oil Induced Environmental Degradation in the Nigeria's Niger Delta: The Multiplier Effects. Journal of Sustainable Development in Africa, 11, 160-175.

[37] Egbe, R.E. and Thompson, D. (2010) Environmental Challenges of Oil Spillage for Families in Oil Producing Communities of the Niger Delta Region. Journal of Home Economics Research, 13, 24-34.

[38] Kadafa, A.A. (2012) Oil Exploration and Spillage in the Niger Delta of Nigeria. Civil and Environmental Research, 2, No. 3.

[39] Ezeala, P. (2003) Nigeria's Threatened Forests. Nature Watch, 3. 
Scientific Research Publishing (SCIRP) is one of the largest Open Access journal publishers. It is currently publishing more than 200 open access, online, peer-reviewed journals covering a wide range of academic disciplines. SCIRP serves the worldwide academic communities and contributes to the progress and application of science with its publication.

Other selected journals from SCIRP are listed as below. Submit your manuscript to us via either submit@scirp.org or Online Submission Portal.
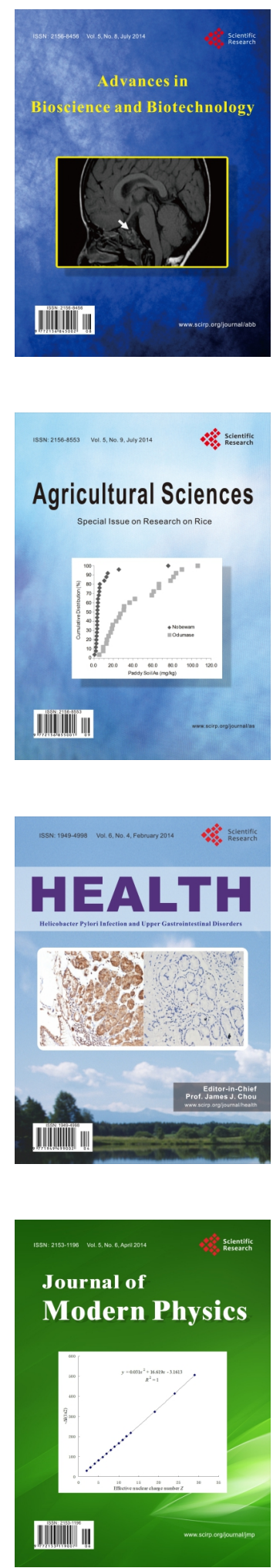
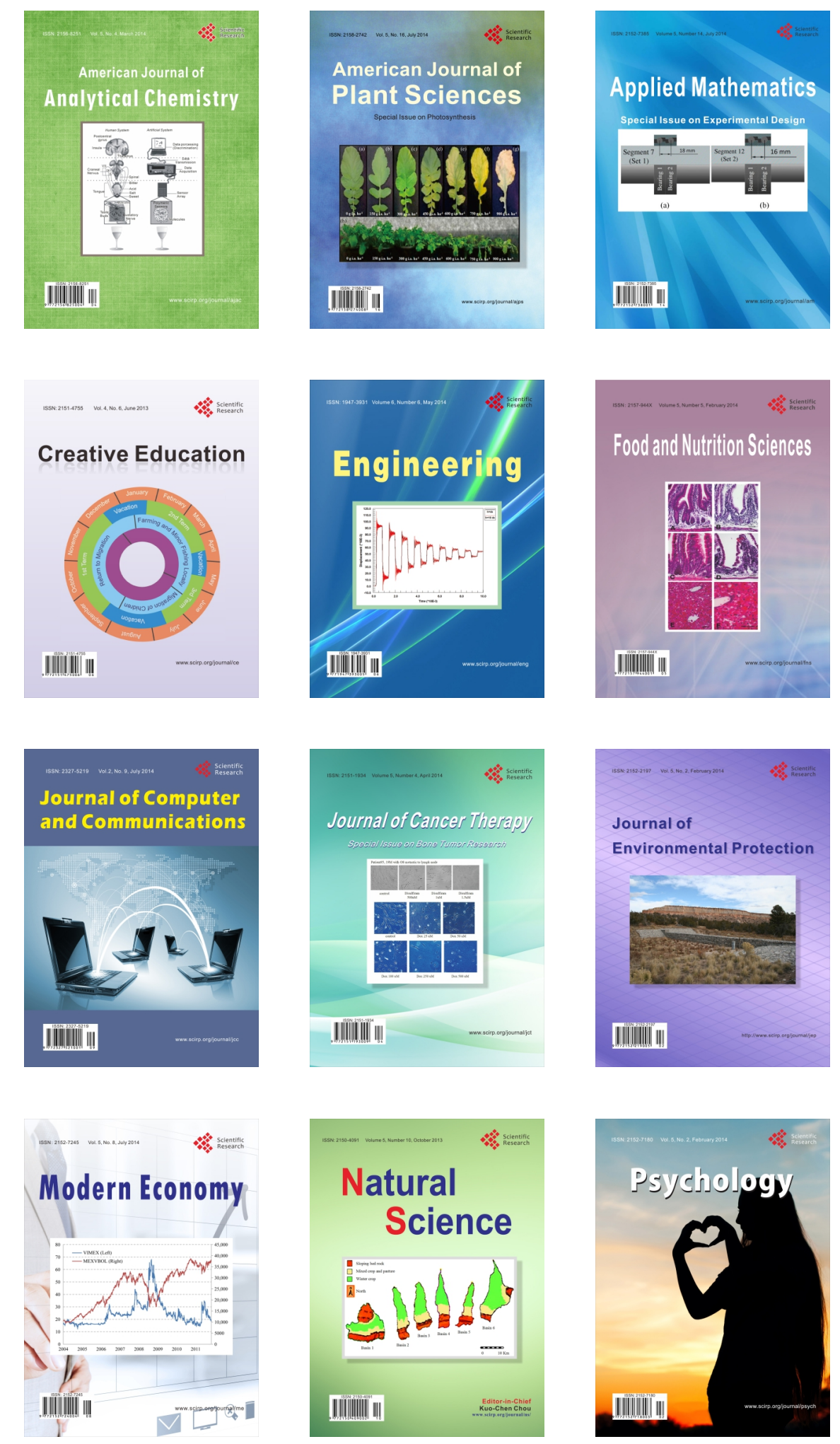